\title{
Universal critical behavior of noisy coupled oscillators: A renormalization group study
}

\author{
Thomas Risler, ${ }^{1,2}$ Jacques Prost, ${ }^{3,2}$ and Frank Jülicher ${ }^{1}$ \\ ${ }^{1}$ Max-Planck-Institut für Physik komplexer Systeme, Nöthnitzerstrasse 38, 01187 Dresden, Germany \\ ${ }^{2}$ Physicochimie Curie (CNRS-UMR 168), Institut Curie, 26 rue d'Ulm, 75248 Paris Cedex 05, France \\ ${ }^{3}$ Ecole Supérieure de Physique et de Chimie Industielles de la Ville de Paris, 10 rue Vauquelin, 75231 Paris Cedex 05, France
}

(Received 17 April 2005; published 29 July 2005)

\begin{abstract}
We show that the synchronization transition of a large number of noisy coupled oscillators is an example for a dynamic critical point far from thermodynamic equilibrium. The universal behaviors of such critical oscillators, arranged on a lattice in a $d$-dimensional space and coupled by nearest-neighbors interactions, can be studied using field-theoretical methods. The field theory associated with the critical point of a homogeneous oscillatory instability (or Hopf bifurcation of coupled oscillators) is the complex Ginzburg-Landau equation with additive noise. We perform a perturbative renormalization group (RG) study in a $(4-\epsilon)$-dimensional space. We develop an RG scheme that eliminates the phase and frequency of the oscillations using a scaledependent oscillating reference frame. Within Callan-Symanzik's RG scheme to two-loop order in perturbation theory, we find that the RG fixed point is formally related to the one of the model $A$ dynamics of the real Ginzburg-Landau theory with an $O(2)$ symmetry of the order parameter. Therefore, the dominant critical exponents for coupled oscillators are the same as for this equilibrium field theory. This formal connection with an equilibrium critical point imposes a relation between the correlation and response functions of coupled oscillators in the critical regime. Since the system operates far from thermodynamic equilibrium, a strong violation of the fluctuation-dissipation relation occurs and is characterized by a universal divergence of an effective temperature. The formal relation between critical oscillators and equilibrium critical points suggests that long-range phase order exists in critical oscillators above two dimensions.
\end{abstract}

DOI: 10.1103/PhysRevE.72.016130

PACS number(s): 64.60.Ht, 05.45.Xt, 64.60.Ak, 05.10.Cc

\section{INTRODUCTION}

Equilibrium systems consisting of a large number of degrees of freedom exhibit phase transitions as a consequence of the collective behavior of many components [1-3]. The universal behaviors near critical points have been studied extensively using field-theoretical methods and renormalization group $(\mathrm{RG})$ techniques [3-13]. In systems driven far from thermodynamic equilibrium, collective behaviors can lead to dynamic instabilities and nonequilibrium phase transitions [14-17]. While the study of nonequilibrium critical points has remained a big challenge, $\mathrm{RG}$ methods have in some cases been applied [18-22].

An important example of nonequilibrium critical behavior is the homogeneous oscillatory instability or Hopf bifurcation of coupled oscillators [23]. Such instabilities are important in many physical, chemical, and biological systems $[24,25]$. From the point of view of statistical physics, phasecoherent oscillations result as the collective behavior of a large number of degrees of freedom in the thermodynamic limit. For a system of finite size, fluctuations destroy the phase coherence of the oscillations, and the singular behaviors characteristic of a Hopf bifurcation are concealed.

This general idea can be illustrated by individual oscillators arranged on a lattice in a $d$-dimensional space and coupled to their nearest neighbors. As a consequence of fluctuations in the system, each oscillator is subject to a noise source. For small coupling strength and as a result of fluctuations, the oscillators have individual phases and exhibit a limited coherence time of oscillations. In the thermodynamic limit, a global phase emerges beyond a critical coupling strength where oscillations become coherent over large dis- tances. As this critical point is approached from the disordered phase, a correlation length, which corresponds to the characteristic size of the domains of synchronized oscillators, diverges. At the same time, oscillations become coherent over long periods of time and long-range phase order appears. The divergence of the correlation length allows a description of the critical behaviors of spatially extended systems in terms of continuous field theories and is characteristic of scale invariance in the critical regime. It constitutes the foundation of the RG theory, which explains the emergence of universality at critical points [4-6,11-13].

In this paper, we perform an RG study of the critical behaviors of a collection of oscillators, distributed on a $d$-dimensional lattice and coupled via nearest-neighbor interactions. We approach the critical point from the disordered phase. Close to criticality, the large-scale properties of the array of oscillators are described by a dynamic field theory that is given by the complex Ginzburg-Landau equation with an additive noise term. We apply field-theoretical perturbation theory in a $d=(4-\epsilon)$-dimensional space and introduce an $\mathrm{RG}$ scheme that is appropriate for the study of coupled oscillators.

The outline of the paper is as follows. In Sec. II, we present the general field-theoretical framework for the complex Ginzburg-Landau equation. We introduce in Sec. III an oscillating reference frame that is essential to define the RG procedure for oscillating critical systems and in which the phase and frequency of the oscillations are eliminated. The correlation and response functions of critical oscillators in mean-field theory are discussed in Sec. IV. These mean-field results are relevant above the critical dimension $d_{c}=4$. For 
$d<4$, mean-field theory breaks down. We discuss in Sec. V the renormalization group of the complex Ginzburg-Landau field theory using Wilson's RG scheme for which the renormalization procedure for critical oscillators can be introduced most clearly. One-loop order calculations in perturbation theory are presented, but further calculations are necessary to characterize the correct qualitative structure of the RG flow. In Sec. VI, we present Callan-Symanzik's RG scheme adapted for systems of coupled oscillators and calculate its beta functions as well as its complete RG flow and fixed points to two-loop order in perturbation theory. The physical properties of the oscillating system are characterized by correlation and response functions. In Sec. VII, we discuss the asymptotic behaviors of these functions in the critical regime using the RG flow and applying a matching procedure. The formal relation of the RG fixed point for critical oscillators to the fixed point of a real GinzburgLandau theory [which satisfies a fluctuation-dissipation (FD) relation] leads to an emergent symmetry at the critical point. However, the system operates far from thermodynamic equilibrium and breaks the FD relation. The degree of this violation can be characterized by the introduction of a frequencydependent effective temperature which diverges with a universal anomalous power law at the critical point. We conclude our presentation with a discussion of the general properties of critical oscillators, their relations to equilibrium critical points, and possible experimental systems for which the critical behaviors discussed here could be observed in the future.

\section{FIELD THEORY OF COUPLED OSCILLATORS}

\section{A. Complex Ginzburg-Landau field theory}

The generic behavior of a nonlinear oscillator in the vicinity of a Hopf bifurcation can be described by a dynamic equation for a complex variable $Z$ characterizing the phase and amplitude of the oscillations [24]. This variable can be chosen such that its real part is, to linear order, related to a physical observable, e.g., the displacement $X(t)$ generated by a mechanical oscillator: $X(t)=\operatorname{Re}[Z(t)]+$ nonlinear terms. In the presence of a periodic stimulus force $F(t)=\widetilde{F} e^{-i \omega t}$ with a frequency $\omega$ close to the oscillation frequency $\omega_{0}$ at the bifurcation, the generic dynamics obeys

$$
\partial_{t} Z=-\left(r+i \omega_{0}\right) Z-\left(u+i u_{a}\right)|Z|^{2} Z+\Lambda^{-1} e^{i \theta} F(t) .
$$

For $F=0$ and $r>0$, the static state $Z=0$ is stable. The system undergoes a Hopf bifurcation at $r=0$ and exhibits spontaneous oscillations for $r<0$. The nonlinear term, characterized by the coefficients $u$ and $u_{a}$, stabilizes the oscillation amplitude for $u>0$. The external stimulus appears linearly in this equation and couples in general with a phase shift $\theta[26,27]$. In the case of a mechanical oscillator, the coefficient $\Lambda$ has units of a friction.

Coupling many oscillators in a field theoretic continuum limit leads to the complex Ginzburg-Landau equation $[28,29]$ with additive noise and external forcing terms,

$$
\begin{aligned}
\partial_{t} Z= & -\left(r+i \omega_{0}\right) Z+\left(c+i c_{a}\right) \Delta Z-\left(u+i u_{a}\right)|Z|^{2} Z \\
& +\Lambda^{-1} e^{i \theta} F+\eta .
\end{aligned}
$$

Here, the complex variable $Z(\mathbf{x}, t)$ becomes a field defined at positions $\mathbf{x}$ in a $d$-dimensional space and $\Delta$ denotes the Laplace operator in this space. The coefficients $c$ and $c_{a}$ characterize the local coupling of oscillators and the effects of fluctuations are described via a complex random forcing term $\eta(\mathbf{x}, t)$, which will be chosen Gaussian with zero mean value, i.e., $\langle\eta(\mathbf{x}, t)\rangle=0$. As far as long-time and longwavelength properties are concerned, the correlations of the noise can be neglected and white noise can be used.

For a vanishing external field $F(\mathbf{x}, t)$ and in the absence of fluctuations, Eq. (2) is invariant with respect to phase changes of the oscillations,

$$
Z \rightarrow Z e^{i \phi} .
$$

This symmetry reflects the fact that only phase-invariant terms contribute to the dominant critical behaviors studied here. Indeed, the Hopf bifurcation is associated with the emergence of a nonzero oscillatory mode, which dominates the critical behaviors and for which time-translational invariance and phase invariance are equivalent. The noise correlations can therefore be chosen such that they respect phase invariance in the problem,

$$
\begin{gathered}
\left\langle\eta(\mathbf{x}, t) \eta\left(\mathbf{x}^{\prime}, t^{\prime}\right)\right\rangle=0, \\
\left\langle\eta(\mathbf{x}, t) \eta^{*}\left(\mathbf{x}^{\prime}, t^{\prime}\right)\right\rangle=4 D \delta^{d}\left(\mathbf{x}-\mathbf{x}^{\prime}\right) \delta\left(t-t^{\prime}\right) .
\end{gathered}
$$

Here $D$ is a real and positive coefficient characterizing the amplitude of the noise, and $\delta$ and $\delta^{d}$ represent Dirac distributions, respectively, in 1 and $d$ dimensions.

\section{B. Physical correlation and response functions}

Since the physical variables of interest are real, we decompose the complex fields $Z$ and $F$ into their real and imaginary parts by $Z=\psi_{1}+i \psi_{2}$ and $F=F_{1}+i F_{2}$. We focus on two important functions which characterize the behavior of the system, namely the two-point autocorrelation function $C_{\alpha \beta}$ and the linear-response function $\chi_{\alpha \beta}$ to an applied external forcing term. They are defined as

$$
\begin{gathered}
C_{\alpha \beta}\left(\mathbf{x}-\mathbf{x}^{\prime}, t-t^{\prime}\right)=\left\langle\psi_{\alpha}(\mathbf{x}, t) \psi_{\beta}\left(\mathbf{x}^{\prime}, t^{\prime}\right)\right\rangle_{c}, \\
\left\langle\psi_{\alpha}(\mathbf{x}, t)\right\rangle=\int d^{d} x^{\prime} d t^{\prime} \chi_{\alpha \beta}\left(\mathbf{x}-\mathbf{x}^{\prime}, t-t^{\prime}\right) F_{\beta}\left(\mathbf{x}^{\prime}, t^{\prime}\right)+O\left(|F|^{2}\right),
\end{gathered}
$$

where $\langle\cdots\rangle_{c}$ denotes a connected correlation function [51]. Because of phase invariance, these functions obey the following symmetry relations:

$$
C_{11}=C_{22} \quad \text { and } \quad C_{21}=-C_{12},
$$

with similar relations for the linear-response function $\chi_{\alpha \beta}$.

\section{Field-theoretical representation}

The correlation and response functions $C_{\alpha \beta}$ and $\chi_{\alpha \beta}$ can be conveniently expressed using a field-theoretical formal- 
ism. We introduce the Martin-Siggia-Rose response field $\widetilde{\psi}_{\alpha}$ [30] and apply the Janssen-De Dominicis formalism [31,32] to write the following generating functional:

$$
\begin{aligned}
Z_{P}\left[\tilde{I}_{\alpha}, I_{\alpha}\right]= & \int \mathcal{D}\left[\psi_{\alpha}\right] \mathcal{D}\left[-i \tilde{\psi}_{\alpha}\right] \\
& \times \exp \left\{\mathcal{S}_{P}\left[\tilde{\psi}_{\alpha}, \psi_{\alpha}\right]+\int d^{d} x d t\left[\tilde{I}_{\alpha} \tilde{\psi}_{\alpha}+I_{\alpha} \psi_{\alpha}\right]\right\},
\end{aligned}
$$

where the "physical" action $\mathcal{S}_{P}$ is given by [52]

$$
\begin{aligned}
\mathcal{S}_{P}\left[\tilde{\psi}_{\alpha}, \psi_{\alpha}\right]= & \int d^{d} x d t\left\{D \tilde{\psi}_{\alpha} \tilde{\psi}_{\alpha}-\tilde{\psi}_{\alpha}\left[\partial_{t} \psi_{\alpha}+\left(R_{\alpha \beta}+\omega_{0} \varepsilon_{\alpha \beta}\right) \psi_{\beta}\right.\right. \\
& \left.\left.+U_{\alpha \beta} \psi_{\beta} \psi_{\gamma} \psi_{\gamma}\right]\right\} .
\end{aligned}
$$

Here $R_{\alpha \beta}=(r-c \Delta) \delta_{\alpha \beta}-c_{a} \Delta \varepsilon_{\alpha \beta}$ and $U_{\alpha \beta}=u \delta_{\alpha \beta}+u_{a} \varepsilon_{\alpha \beta}$, with $\varepsilon_{21}=-\varepsilon_{12}=1$ and $\varepsilon_{11}=\varepsilon_{22}=0$. The field $\tilde{I}_{\alpha}$ is related to the external force $F_{\alpha}$ in the equation of motion [Eq. (2)] by

$$
\tilde{I}_{\alpha}=\Lambda^{-1} \Omega_{\alpha \beta}(\theta) F_{\beta},
$$

where $\Omega_{\alpha \beta}(\theta)$ denotes the rotation matrix by an angle $\theta$ in two dimensions,

$$
\Omega_{\alpha \beta}(\theta)=\left(\begin{array}{cc}
\cos (\theta) & -\sin (\theta) \\
\sin (\theta) & \cos (\theta)
\end{array}\right) .
$$

Correlation and response functions are given by derivatives of the generating functional. We have

$$
\begin{gathered}
C_{\alpha \beta}\left(\mathbf{x}-\mathbf{x}^{\prime}, t-t^{\prime}\right)=\left.\frac{\delta^{2} \ln Z}{\delta I_{\alpha}(\mathbf{x}, t) \delta I_{\beta}\left(\mathbf{x}^{\prime}, t^{\prime}\right)}\right|_{I_{\alpha}, \tilde{I}_{\alpha}=0}, \quad(11) \\
\chi_{\alpha \beta}\left(\mathbf{x}-\mathbf{x}^{\prime}, t-t^{\prime}\right)=\left.\Lambda^{-1} \Omega_{\gamma \beta}(\theta) \frac{\delta^{2} \ln Z}{\delta I_{\alpha}(\mathbf{x}, t) \delta \tilde{I}_{\gamma}\left(\mathbf{x}^{\prime}, t^{\prime}\right)}\right|_{I_{\alpha}, \tilde{I}_{\alpha}=0} .
\end{gathered}
$$

\section{FIELD THEORY IN AN OSCILLATING REFERENCE FRAME}

\section{A. Amplitude equation}

The frequency $\omega_{0}$ and the phase $\theta$ can be eliminated from Eq. (2) by a time-dependent variable transformation of the form $Y \equiv e^{i \omega_{0} t} Z, H \equiv e^{i \omega_{0} t} \Lambda^{-1} e^{i \theta} F$, and $\zeta \equiv e^{i \omega_{0} t} \eta$, where $Y$ denotes the oscillation amplitude, $H$ is a forcing amplitude, and $\zeta$ is a transformed noise that has the same correlators as $\eta$. This procedure leads to the amplitude equation

$$
\partial_{t} Y=-r Y+\left(c+i c_{a}\right) \Delta Y-\left(u+i u_{a}\right)|Y|^{2} Y+H+\zeta .
$$

Defining two real fields $\phi_{\alpha}$ by $Y=\phi_{1}+i \phi_{2}$, Eq. (13) reads

$$
\partial_{t} \phi_{\alpha}=-R_{\alpha \beta} \phi_{\beta}-U_{\alpha \beta} \phi_{\beta} \phi_{\gamma} \phi_{\gamma}+H_{\alpha}+\zeta_{\alpha},
$$

where $H=H_{1}+i H_{2}$.

The correlation and response functions $G_{\alpha \beta}=\left\langle\phi_{\alpha} \phi_{\beta}\right\rangle_{c}$ and $\gamma_{\alpha \beta}=\left\langle\phi_{\alpha} \widetilde{\phi}_{\beta}\right\rangle_{c}$ of the fields $\phi_{\alpha}$ are related to the physical correlation and response functions by

$$
\begin{gathered}
C_{\alpha \beta}(\mathbf{x}, t)=\Omega_{\alpha \sigma}\left(-\omega_{0} t\right) G_{\sigma \beta}(\mathbf{x}, t), \\
\chi_{\alpha \beta}(\mathbf{x}, t)=\Lambda^{-1} \Omega_{\alpha \sigma}\left(\theta-\omega_{0} t\right) \gamma_{\sigma \beta}(\mathbf{x}, t) .
\end{gathered}
$$

Similar time-dependent transformations exist between higher-order correlation and response functions of the physical fields and those calculated in the oscillating reference frame.

\section{B. Analogy with an equilibrium critical point in a particular case}

For the particular case where $c_{a}=0$ and $u_{a}=0$, Eq. (14) becomes identical to the model A dynamics of a real Ginzburg-Landau field theory with an $O(2)$ symmetry of the order parameter [3]. The critical behavior of this theory at thermodynamic equilibrium has been extensively studied [33-35]. This leads, in this particular case, to a formal analogy between an equilibrium phase transition and a Hopf bifurcation. The amplitude $Y$ plays the role of the order parameter of the transition. The disordered phase with $\langle Y\rangle=0$ corresponds to noisy oscillators that are not in synchrony, while nonzero order $\langle Y\rangle$ implies the existence of a global phase and amplitude of synchronous oscillations with frequency $\omega_{0}$. The correlation lengths and times of the equilibrium field theory correspond to lengths and times over which oscillators are in synchrony. The correlation and response functions $C_{\alpha \beta}$ and $\chi_{\alpha \beta}$ for this case can be obtained from those of the equilibrium field theory by using Eq. (15). Since the $O(2)$ symmetric theory at thermodynamic equilibrium obeys an FD relation, a generic relation between the correlation and response functions $C_{\alpha \beta}$ and $\chi_{\alpha \beta}$ appears.

\section{Generating functional}

The functions $G_{\alpha \beta}$ and $\gamma_{\alpha \beta}$, as well as higher-order correlation and response functions, can be formally calculated using field-theoretical techniques (see, e.g., $[2,13])$. The generating functional of the theory is given by

$$
\begin{aligned}
Z\left[\tilde{J}_{\alpha}, J_{\alpha}\right]= & \int \mathcal{D}\left[\phi_{\alpha}\right] \mathcal{D}\left[-i \widetilde{\phi}_{\alpha}\right] \\
& \times \exp \left\{\mathcal{S}\left[\tilde{\phi}_{\alpha}, \phi_{\alpha}\right]+\int d^{d} x d t\left[\tilde{J}_{\alpha} \tilde{\phi}_{\alpha}+J_{\alpha} \phi_{\alpha}\right]\right\},
\end{aligned}
$$

where we have introduced the Martin-Siggia-Rose response field $\widetilde{\phi}_{\alpha}[30]$. The associated action reads

$$
\begin{aligned}
\mathcal{S}\left[\widetilde{\phi}_{\alpha}, \phi_{\alpha}\right]= & \int d^{d} x d t\left\{D \widetilde{\phi}_{\alpha} \widetilde{\phi}_{\alpha}-\widetilde{\phi}_{\alpha}\left[\partial_{t} \phi_{\alpha}+R_{\alpha \beta} \phi_{\beta}\right]\right. \\
& \left.-U_{\alpha \beta} \widetilde{\phi}_{\alpha} \phi_{\beta} \phi_{\gamma} \phi_{\gamma}\right\} .
\end{aligned}
$$

Correlation and response functions are given by

$$
G_{\alpha \beta}\left(\mathbf{x}-\mathbf{x}^{\prime}, t-t^{\prime}\right)=\left.\frac{\delta^{2} \ln Z}{\delta J_{\alpha}(\mathbf{x}, t) \delta J_{\beta}\left(\mathbf{x}^{\prime}, t^{\prime}\right)}\right|_{J_{\alpha^{\prime}} \tilde{J}_{\alpha}=0},
$$




$$
\gamma_{\alpha \beta}\left(\mathbf{x}-\mathbf{x}^{\prime}, t-t^{\prime}\right)=\left.\frac{\delta^{2} \ln Z}{\delta J_{\alpha}(\mathbf{x}, t) \delta \widetilde{J}_{\beta}\left(\mathbf{x}^{\prime}, t^{\prime}\right)}\right|_{J_{\alpha^{\prime}} \tilde{J}_{\alpha}=0} .
$$

The effective action of the theory is defined by

$$
\Gamma\left[\widetilde{\Phi}_{\alpha}, \Phi_{\alpha}\right]=\int d^{d} x d t\left[\widetilde{J}_{\alpha} \widetilde{\Phi}_{\alpha}+J_{\alpha} \Phi_{\alpha}\right]-\ln Z\left[\widetilde{J}_{\alpha}, J_{\alpha}\right],
$$

with

$$
\Phi_{\alpha}(\mathbf{x}, t)=\frac{\delta \ln Z}{\delta J_{\alpha}(\mathbf{x}, t)} \quad \text { and } \quad \widetilde{\Phi}_{\alpha}(\mathbf{x}, t)=\frac{\delta \ln Z}{\delta \widetilde{J}_{\alpha}(\mathbf{x}, t)} .
$$

In order to perform calculations perturbatively, we split the action into a harmonic or "Gaussian" part, and a quartic or "interaction" part as $\mathcal{S}=\mathcal{S}_{0}+\mathcal{S}_{\text {int }}$. $\mathcal{S}_{0}$ is given by

$$
\begin{aligned}
\mathcal{S}_{0} & =\int_{k}\left(\widetilde{\phi}_{\alpha}(k)\left\{D \widetilde{\phi}_{\alpha}(-k)-\left[i \omega \delta_{\alpha \beta}+R_{\alpha \beta}(-k)\right] \phi_{\beta}(-k)\right\}\right) \\
& =-\frac{1}{2} \int_{k} \underline{\phi}_{\alpha}^{t}(k) \underline{A}_{\alpha \beta}(-k) \underline{\phi}_{\beta}(-k)
\end{aligned}
$$

where

$$
\begin{gathered}
\underline{\phi}_{\alpha}=\left(\begin{array}{c}
\widetilde{\phi}_{\alpha} \\
\phi_{\alpha}
\end{array}\right), \quad \underline{\phi}_{\alpha}^{t}=\left(\widetilde{\phi}_{\alpha}, \phi_{\alpha}\right), \\
\underline{A}_{\alpha \beta}(-k)=\left(\begin{array}{cc}
-2 D \delta_{\alpha \beta} & i \omega \delta_{\alpha \beta}+R_{\alpha \beta}(-k) \\
-i \omega \delta_{\beta \alpha}+R_{\beta \alpha}(k) & 0
\end{array}\right),
\end{gathered}
$$

and $R_{\alpha \beta}(k)=\left(r+c \mathbf{q}^{2}\right) \delta_{\alpha \beta}+c_{a} \mathbf{q}^{2} \varepsilon_{\alpha \beta}$. In these expressions and in the following, we label $k=(\mathbf{q}, \omega), \int_{k}=\int_{\mathbf{q}, \omega}$ denotes $\int\left[d^{d} q /(2 \pi)^{d}\right](d \omega / 2 \pi)$, and we use the following convention for Fourier-transforms:

$$
f(\mathbf{x}, t)=\int_{\mathbf{q}, \omega} f(\mathbf{q}, \omega) e^{i(\mathbf{q} \cdot \mathbf{x}-\omega t)}=\int_{k} f(k) e^{i k \cdot x} .
$$

The interaction term of the action takes the form

$$
\begin{aligned}
\mathcal{S}_{\text {int }}= & -\int_{\left\{k_{i}\right\}} U_{\alpha \beta} \widetilde{\phi}_{\alpha}\left(k_{1}\right) \phi_{\beta}\left(k_{2}\right) \phi_{\gamma}\left(k_{3}\right) \phi_{\gamma}\left(k_{4}\right) \\
& \times(2 \pi)^{d+1} \delta^{(d+1)}\left(\sum_{i} k_{i}\right) .
\end{aligned}
$$

Graphic representations of the basic diagrams of the perturbation theory are given in Appendix A.

\section{MEAN-FIELD THEORY}

Dimensional analysis reveals that for $d>4$ mean-field theory applies. In this case, the mean-field approximation allows us to calculate valid asymptotic expressions for the effective action of the theory, as well as the two-point correlation and response functions. In the framework of the Janssen-De Dominicis formalism for dynamic field- theoretical models, this approximation consists in substituting the saddle-node value of the path integral (16) to the full functional generator $Z$. We obtain

$$
Z^{\mathrm{mf}[}\left[\widetilde{J}_{\alpha}, J_{\alpha}\right]=\exp \left\{\mathcal{S}\left[\widetilde{\phi}_{\alpha}^{\mathrm{mf}}, \phi_{\alpha}^{\mathrm{mf}}\right]+\int d^{d} x d t\left[\widetilde{J}_{\alpha} \widetilde{\phi}_{\alpha}^{\mathrm{mf}}+J_{\alpha} \phi_{\alpha}^{\mathrm{mf}}\right]\right\},
$$

where $\mathcal{S}$ is given by Eq. (17) and $\phi_{\alpha}^{\mathrm{mf}}$ and $\widetilde{\phi}_{\alpha}^{\mathrm{mf}}$ satisfy the stationarity conditions at the saddle node

$$
\frac{\delta \mathcal{S}}{\delta \widetilde{\phi}_{\alpha}}=-\widetilde{J}_{\alpha} \quad \text { and } \quad \frac{\delta \mathcal{S}}{\delta \phi_{\alpha}}=-J_{\alpha},
$$

which give the mean-field dynamic equations

$$
\begin{gathered}
J_{\alpha}=-\partial_{t} \widetilde{\phi}_{\alpha}^{\mathrm{mf}}+R_{\beta \alpha} \widetilde{\phi}_{\beta}^{\mathrm{mf}}+U_{\beta \alpha} \widetilde{\phi}_{\beta}^{\mathrm{mf}} \phi_{\gamma}^{\mathrm{mf}} \phi_{\gamma}^{\mathrm{mf}}+2 U_{\beta \sigma} \phi_{\sigma}^{\mathrm{mf}} \phi_{\alpha}^{\mathrm{mf}} \widetilde{\phi}_{\beta}^{\mathrm{mf}}, \\
\widetilde{J}_{\alpha}=-2 D \widetilde{\phi}_{\alpha}^{\mathrm{mf}}+\left[\partial_{t} \phi_{\alpha}^{\mathrm{mf}}+R_{\alpha \beta} \phi_{\beta}^{\mathrm{mf}}+U_{\alpha \beta} \phi_{\beta}^{\mathrm{mf}} \phi_{\gamma}^{\mathrm{mf}} \phi_{\gamma}^{\mathrm{mf}}\right] .
\end{gathered}
$$

The correlation and response functions can be obtained most easily by first eliminating the nonphysical field $\widetilde{\phi}_{\alpha}^{\mathrm{mf}}$ and writing a mean-field equation for $\phi_{\alpha}^{\mathrm{mf}}$ only,

$$
\begin{aligned}
2 D J_{\alpha}= & {\left[-\partial_{t} \delta_{\alpha \beta}+R_{\beta \alpha}+U_{\beta \alpha} \phi_{\gamma}^{\mathrm{mf}} \phi_{\gamma}^{\mathrm{mf}}+2 U_{\beta \sigma} \phi_{\sigma}^{\mathrm{mf}} \phi_{\alpha}^{\mathrm{mf}}\right] } \\
& \times\left[\partial_{t} \phi_{\beta}^{\mathrm{mf}}+R_{\beta \sigma} \phi_{\sigma}^{\mathrm{mf}}+U_{\beta \sigma} \phi_{\sigma}^{\mathrm{mf}} \phi_{\gamma}^{\mathrm{mf}} \phi_{\gamma}^{\mathrm{mf}}-\widetilde{J}_{\beta}\right] .
\end{aligned}
$$

The mean-field generating functional for the field $\phi_{\alpha}^{\mathrm{mf}}$ obeying Eq. (30) is obtained from Eq. (27) by eliminating the field $\widetilde{\phi}_{\alpha}^{\mathrm{mf}}$. It reads

$$
\begin{aligned}
Z^{\mathrm{mf} f}\left[\tilde{J}_{\alpha}, J_{\alpha}\right]= & \exp \left\{-\frac{1}{4 D} \int d^{d} x d t\left[\left(\partial_{t} \phi_{\alpha}^{\mathrm{mf}}-F_{\alpha}\left[\phi_{\beta}^{\mathrm{mf}}\right]-\tilde{J}_{\alpha}\right)^{2}\right.\right. \\
& \left.\left.+J_{\alpha} \phi_{\alpha}^{\mathrm{mf}}\right]\right\}
\end{aligned}
$$

where

$$
F_{\alpha}\left[\phi_{\beta}\right]=-R_{\alpha \beta} \phi_{\beta}-U_{\alpha \beta} \phi_{\beta} \phi_{\gamma} \phi_{\gamma} .
$$

Note that for $J_{\alpha}=0$ the stationary condition of the generating functional (31) leads to

$$
\partial_{t} \phi_{\alpha}^{\mathrm{mf}}=-R_{\alpha \beta} \phi_{\beta}^{\mathrm{mf}}-U_{\alpha \beta} \phi_{\beta}^{\mathrm{mf}} \phi_{\gamma}^{\mathrm{mf}} \phi_{\gamma}^{\mathrm{mf}}+\widetilde{J}_{\alpha},
$$

which is compatible with Eq. (30).

The linear-response and correlation functions are obtained as

$$
\begin{aligned}
& \gamma_{\alpha \beta}^{\mathrm{mf}}\left(\mathbf{x}-\mathbf{x}^{\prime} ; t-t^{\prime}\right)=\left.\frac{\delta \phi_{\alpha}^{\mathrm{mf}}(\mathbf{x}, t)}{\delta \widetilde{J}_{\beta}\left(\mathbf{x}^{\prime}, t^{\prime}\right)}\right|_{J_{\alpha}, \tilde{J}_{\alpha} \equiv 0}, \\
& G_{\alpha \beta}^{\mathrm{mf}}\left(\mathbf{x}-\mathbf{x}^{\prime} ; t-t^{\prime}\right)=\left.\frac{\delta \phi_{\alpha}^{\mathrm{mf}}(\mathbf{x}, t)}{\delta J_{\beta}\left(\mathbf{x}^{\prime}, t^{\prime}\right)}\right|_{J_{\alpha}, \tilde{J}_{\alpha} \equiv 0} .
\end{aligned}
$$

Calculating these functions and applying the time-dependent transformations (15), we obtain the physical correlation and response functions in mean-field theory, 


$$
\begin{aligned}
\chi_{\alpha \beta}^{\mathrm{mf}}(\mathbf{q}, \omega)= & \frac{1}{\Lambda \Delta}\left\{\left[(-i \omega+R) \cos \theta+\Omega_{0} \sin \theta\right] \delta_{\alpha \beta}\right. \\
& \left.+\left[(-i \omega+R) \sin \theta-\Omega_{0} \cos \theta\right] \varepsilon_{\alpha \beta}\right\} \\
C_{\alpha \beta}^{\mathrm{mf}}(\mathbf{q}, \omega)= & \frac{2 D}{|\Delta|^{2}}\left(\begin{array}{cc}
\omega^{2}+\Omega_{0}^{2}+R^{2} & 2 i \omega \Omega_{0} \\
-2 i \omega \Omega_{0} & \omega^{2}+\Omega_{0}^{2}+R^{2}
\end{array}\right),
\end{aligned}
$$

where $R=r+c \mathbf{q}^{2}, \Omega_{0}=\omega_{0}+c_{a} \mathbf{q}^{2}$, and $\Delta=(-i \omega+R)^{2}+\Omega_{0}^{2}$. The diagonal elements of these matrices are given by

$$
\begin{gathered}
\chi_{11}^{\mathrm{mf}}(\mathbf{q}, \omega)=\frac{1}{2 \Lambda}\left[\frac{e^{i \theta}}{R-i\left(\omega-\Omega_{0}\right)}+\frac{e^{-i \theta}}{R-i\left(\omega+\Omega_{0}\right)}\right], \\
C_{11}^{\operatorname{mf}}(\mathbf{q}, \omega)=\frac{D}{R^{2}+\left(\omega-\Omega_{0}\right)^{2}}+\frac{D}{R^{2}+\left(\omega+\Omega_{0}\right)^{2}},
\end{gathered}
$$

and the nondiagonal elements by

$$
\begin{gathered}
\chi_{12}^{\operatorname{mf}}(\mathbf{q}, \omega)=\frac{i}{2 \Lambda}\left[\frac{e^{i \theta}}{R-i\left(\omega-\Omega_{0}\right)}-\frac{e^{-i \theta}}{R-i\left(\omega+\Omega_{0}\right)}\right], \\
C_{12}^{\operatorname{mf}}(\mathbf{q}, \omega)=\frac{i D}{R^{2}+\left(\omega-\Omega_{0}\right)^{2}}-\frac{i D}{R^{2}+\left(\omega+\Omega_{0}\right)^{2}} .
\end{gathered}
$$

Note finally that for $r=0$ and for the critical mode (q $=\mathbf{0}, \omega=0)$, the response function of the system is nonlinear even at small amplitudes. We have indeed in this case

$$
\delta\langle Y(0,0) \mid\rangle^{\mathrm{mf}} \propto|H|^{1 / 3} .
$$

\section{WILSON'S RENORMALIZATION SCHEME}

For $d<4$, mean-field theory breaks down and another approach is necessary to investigate the critical behaviors of the theory. We apply perturbative renormalization group (RG) methods using an $\epsilon$ expansion near the upper critical dimension $d_{c}=4(d=4-\epsilon)[7,36,37]$. We present here the RG structure of the theory within Wilson's momentum shell RG scheme adapted to the renormalization of the complex Ginzburg-Landau field theory. This scheme has the advantage to be conceptually transparent and to provide a clear physical interpretation to the calculations. However, for calculations beyond one-loop order, this technique is less suited than the Callan-Symanzik RG scheme. The adaptation of the latter to the complex Ginzburg-Landau theory is presented in Sec. VI.

\section{A. Renormalized fields}

The renormalization procedure within Wilson's scheme is performed as follows. We start from a dynamic functional of the theory with a small distance cutoff $\Lambda$ in the integrals over wave vectors. This cutoff corresponds to an underlying lattice of mesh size $a \simeq 2 \pi / \Lambda$. We interpret this as a microscopic theory with an action of the form (8), and associated quantities are labeled with a superscript " 0 ." We calculate the effective action in an oscillating reference frame at a given scale $\Lambda / b$, where $b=e^{l}$ is a dilatation coefficient larger than
1. This reference frame is defined such that, described in terms of effective or renormalized parameters, the effective action has the structure (17). The renormalized quantities that satisfy this requirement can be expressed as

$$
\begin{gathered}
\mathbf{q}=b \mathbf{q}^{0}, \quad \omega=b^{2} Z_{\omega}(b) \omega^{0}, \\
\phi_{\alpha}(\mathbf{q}, t)=b^{-(d+2) / 2} \sqrt{Z_{\phi}(b)} Z_{\omega}(b) \Omega_{\alpha \beta}\left(\hat{\omega}_{0}(b) t\right) \psi_{\beta}^{0}\left(\mathbf{q}^{0}, t^{0}\right), \\
\widetilde{\phi}_{\alpha}(\mathbf{q}, t)=b^{-(d-2) / 2} \sqrt{Z_{\tilde{\phi}}(b)} Z_{\omega}(b) \Omega_{\alpha \beta}\left(\theta(b)+\hat{\omega}_{0}(b) t\right) \widetilde{\psi}_{\beta}^{0}\left(\mathbf{q}^{0}, t^{0}\right) .
\end{gathered}
$$

Here we have introduced scale-dependent $Z$ factors for the renormalization of the two dynamic fields $\left[Z_{\phi}(b)\right.$ and $\left.Z_{\widetilde{\phi}}(b)\right]$ and for the frequencies $\left[Z_{\omega}(b)\right]$. In addition to the usual RG transformations and scale dilatation $\mathbf{q}=b \mathbf{q}^{(0)}$, the complex Ginzburg-Landau theory requires us to perform timedependent transformations between bare and renormalized fields. Indeed, the effective theory is described in a reference frame that oscillates with effective frequency $\hat{\omega}_{0}(b)$ and phase $\theta(b)$ relative to the reference frame of the bare theory. The definition of the fields $\phi_{\alpha}$ and $\widetilde{\phi}_{\alpha}$ takes this relative rotation into account by terms involving the rotation matrix $\Omega_{\alpha \beta}$ [Eq. (10)]. The scale-dependent oscillating reference frame represents a key element of the RG procedure for oscillating systems. For the microscopic theory, $\hat{\omega}_{0}(1)=\omega_{0}^{0}$, $\theta(1)=0$ and the fields $\psi_{\alpha}^{0}$ and $\widetilde{\psi}_{\alpha}^{0}$ coincide with the fields introduced in Sec. II.

\section{B. Renormalization group flow}

In order to determine the behavior of the effective parameters under renormalization, we determine their variations with respect to a small changes of the dilatation $\delta b / b$ (or $\delta l$ ). Integrating over the momentum shell of wave vectors in the interval $\left[\Lambda / e^{\delta l}, \Lambda\right]$, we obtain an effective action that, before rescaling, reads

$$
\begin{aligned}
\mathcal{S}^{(1)}\left[\widetilde{\phi}_{\alpha}, \phi_{\alpha}\right]= & \int_{\mathbf{q}}^{\Lambda / e^{\delta l}} \int_{\omega}\left\{(D+\delta D) \widetilde{\phi}_{\alpha} \widetilde{\phi}_{\alpha}\right. \\
& -\widetilde{\phi}_{\alpha}\left[i\left(1+\delta \lambda_{1}\right) \omega+(r+\delta r)+(c+\delta c) \mathbf{q}^{2}\right] \phi_{\alpha} \\
& \left.-\widetilde{\phi}_{\alpha}\left[i \delta \lambda_{2} \omega+\delta \hat{\omega}_{0}+\left(c_{a}+\delta c_{a}\right) \mathbf{q}^{2}\right] \varepsilon_{\alpha \beta} \phi_{\beta}\right\} \\
& -\int_{k_{1} k_{2} k_{3}}^{\Lambda / e^{\delta l}}\left(U_{\alpha \beta}+\delta U_{\alpha \beta}\right) \widetilde{\phi}_{\alpha} \phi_{\beta} \phi_{\gamma} \phi_{\gamma} .
\end{aligned}
$$

Here, we have introduced variations of the parameters under this procedure, which can be calculated perturbatively from the "one-particle irreducible" (1-PI) diagrams of the theory. Other terms are either forbidden by symmetry properties of the theory or irrelevant in the infrared (IR) limit. Two new terms appear, which were absent from Eq. (17) and which correspond to the coefficients $\delta \lambda_{2}$ and $\delta \hat{\omega}_{0}$. They reflect the renormalization of frequency and phase. These terms are absorbed by time-dependent variable transformations of the fields, 


$$
\begin{gathered}
\phi_{\alpha}^{\mathrm{i}}(\mathbf{q}, t)=\Omega_{\alpha \beta}\left(\delta \hat{\omega}_{0}^{\mathrm{i}} t\right) \phi_{\beta}(\mathbf{q}, t), \\
\widetilde{\phi}_{\alpha}^{\mathrm{i}}(\mathbf{q}, t)=\Omega_{\alpha \beta}\left(\delta \hat{\omega}_{0}^{\mathrm{i}} t+\delta \theta\right) \phi_{\beta}(\mathbf{q}, t),
\end{gathered}
$$

where

$$
\begin{gathered}
\delta \theta=\arctan \left[-\delta \lambda_{2}\left(1+\delta \lambda_{1}\right)^{-1}\right], \\
\delta \hat{\omega}_{0}^{\mathrm{i}}=\delta \hat{\omega}_{0} \cos \delta \theta+(r+\delta r) \sin \delta \theta .
\end{gathered}
$$

Rewriting the effective action in terms of the new fields $\phi_{\alpha}^{i}$ and $\widetilde{\phi}_{\alpha}^{i}$ requires a redefinition of the changes of all parameters. The so defined parameter changes are label by a superscript " $i$ " in the following. We finally rescale all lengths by $\mathbf{q}^{\prime}=e^{\delta l} \mathbf{q}$, and introduce three $Z$ factors at the scale $l+\delta l$, such that the effective action retains its form (17) under an RG step. Furthermore, we impose that $D(l+\delta l)=D(l)$ and $c(l+\delta l)=c(l)$, i.e., that the coefficients $c$ and $D$ remain constants under renormalization. As a result, the variations of the parameters under a small renormalization step are given by

$$
\begin{gathered}
c_{a}(l+\delta l)=\left[c_{a}(l)+\delta c_{a}^{\mathrm{i}}\right]\left(1+\delta c^{\mathrm{i}} / c\right)^{-1}, \\
r(l+\delta l)=e^{2 \delta l[}\left[r(l)+\delta r^{\mathrm{i}}\right]\left(1+\delta c^{\mathrm{i}} / c\right)^{-1}, \\
U_{\alpha \beta}(l+\delta l)=e^{(4-d) \delta l}\left[U_{\alpha \beta}(l)+\delta U_{\alpha \beta}^{\mathrm{i}}\right]\left(1+\delta c^{\mathrm{i}} / c\right)^{-2}\left(1+\delta D^{\mathrm{i}} / D\right) \\
\times\left(1+\delta \lambda^{\mathrm{i}}\right)^{-1}, \\
Z_{\omega}(l+\delta l)=Z_{\omega}(l)\left(1+\delta c^{\mathrm{i}} / c\right)^{-1}\left(1+\delta \lambda^{\mathrm{i}}\right), \\
Z_{\phi}(l+\delta l)=Z(l)\left(1+\delta c^{\mathrm{i}} / c\right)^{3}\left(1+\delta D^{\mathrm{i}} / D\right)^{-1}\left(1+\delta \lambda^{\mathrm{i}}\right)^{-1}, \\
Z_{\tilde{\phi}}(l+\delta l)=\tilde{Z}(l)\left(1+\delta c^{\mathrm{i}} / c\right)\left(1+\delta D^{\mathrm{i}} / D\right)\left(1+\delta \lambda^{\mathrm{i}}\right)^{-1},,
\end{gathered}
$$

where

$$
\begin{gathered}
\delta D^{\mathrm{i}}=\delta D, \quad \delta \lambda^{\mathrm{i}}=(C-1)+\delta \lambda_{1} C-\delta \lambda_{2} S, \\
\delta r^{\mathrm{i}}=r(C-1)+\delta r C-\left(\omega_{0}+\delta \omega_{0}\right) S, \\
\delta c^{\mathrm{i}}=c(C-1)+\delta c C-\left(c_{a}+\delta c_{a}\right) S, \\
\delta c_{a}^{\mathrm{i}}=c_{a}(C-1)+\delta c_{a} C+(c+\delta c) S, \\
\delta U_{\alpha \beta}^{\mathrm{i}}=U_{\alpha \sigma}\left(\Omega_{\sigma \beta}(\delta \theta)-I_{\sigma \beta}\right)+\delta U_{\alpha \sigma} \Omega_{\sigma \beta}(\delta \theta) .
\end{gathered}
$$

Here, $C$ and $S$ denote $\cos \delta \theta$ and $\sin \delta \theta$, respectively. The evolution of the effective frequency and phase that enter the transformations (39) is given by

$$
\begin{gathered}
\theta(l+\delta l)=\theta(l)+\delta \theta, \\
\hat{\omega}_{0}(l+\delta l)=e^{2 \delta l}\left[\hat{\omega}_{0}(l)+\delta \hat{\omega}_{0}^{\mathrm{i}}\right]\left(1+\delta c^{\mathrm{i}} / c\right)^{-1}\left(1+\delta \lambda^{\mathrm{i}}\right) .
\end{gathered}
$$

\section{Correlation and response functions}

Using the RG transformations of the effective parameters, as well the correlation and response functions of the renor- malized fields $\phi_{\alpha}$ and $\widetilde{\phi}_{\alpha}$, we can write expressions for the physical correlation and response functions, where the transformations (39) have been used,

$$
\begin{gathered}
C_{\alpha \beta}(\mathbf{q}, t)=b^{2} Z_{\phi}(b)^{-1} Z_{\omega}(b)^{-2} \Omega_{\alpha \sigma}\left(-\omega_{0}(b) t\right) \\
\times G_{\sigma \beta}^{\mathrm{R}}\left(b \mathbf{q}, b^{-2} Z_{\omega}(b)^{-1} t\right), \\
\chi_{\alpha \beta}(\mathbf{q}, t)=\left[Z_{\phi}(b) Z_{\tilde{\phi}}(b)\right]^{-1 / 2} Z_{\omega}(b)^{-2} \Omega_{\alpha \sigma}\left(\theta(b)-\omega_{0}(b) t\right) \\
\times \gamma_{\sigma \beta}^{\mathrm{R}}\left(b \mathbf{q}, b^{-2} Z_{\omega}(b)^{-1} t\right) .
\end{gathered}
$$

Here, the superscript " $R$ " indicates that the functions have to be calculated using the renormalized set of parameters. The scale-dependent renormalized frequency is given by

$$
\omega_{0}(b)=b^{-2} Z_{\omega}(b)^{-1} \hat{\omega}_{0}(b),
$$

and $\theta(b)$ describes the scale-dependent phase lag between external forcing and response of the system.

At the fixed point of the RG, the theory is scale invariant and, consequently, the $Z$ factors exhibit simple scaling relations as a function of $b$,

$$
\begin{gathered}
Z_{\omega}(b)=b^{z-2}, \quad Z_{\phi}(b)=b^{-2(z-2)+\eta}, \\
Z_{\tilde{\phi}}(b)=b^{-2(z-2)+\tilde{\eta}} .
\end{gathered}
$$

These relations define three independent critical exponents of the theory: $z, \eta$, and $\widetilde{\eta}$. A further critical exponent $\nu$ is associated with the positive eigenvalue of the linearized RG equations around the fixed point.

\section{Renormalization of the time and independent critical exponents}

In dynamic $\mathrm{RG}$ procedures, there is in general a freedom to choose some parameters constant while others are renormalized in a nontrivial way. In the system described here, we choose to renormalize the time (i.e., the frequency coordinate $\omega$ in Fourier space) and to keep the parameters $c$ and $D$ invariant under renormalization. In other cases, different choices are commonly used. For example, renormalizing the dynamic model A with $O(2)$ symmetry at thermodynamic equilibrium, the coefficient $D$ is usually chosen to change under renormalization, while time is simply rescaled [34,35]. This model corresponds to the particular case of the amplitude equation of the complex Ginzburg-Landau theory where both $c_{a}$ and $u_{a}$ are equal to zero. It is described by a dynamics of the form

$$
\partial_{t} \phi_{\alpha}=-D \frac{\delta \mathcal{H}}{\delta \phi_{\alpha}}+\zeta_{\alpha},
$$

and relaxes towards a thermodynamic equilibrium. Note that since this model satisfies an FD relation, the noise strength $D$ appears as the mobility coefficient in the dynamics. Equation (49) shows that both choices, renormalizing the time or renormalizing $D$, are equivalent. The complex GinzburgLandau theory discussed here does not obey an FD relation. Therefore, a factorization of the coefficient $D$ as it is done in Eq. (49) would be artificial in this case, and would require a 
redefinition of all other parameters. Without this factorization, the structure of the theory requires one to renormalize the time if the coefficient $c$ is kept constant.

Note also that the absence of an FD relation in the theory changes the structure of the RG equations as compared to an equilibrium $O(2)$ model. Indeed, the FD relation imposes a constraint on the renormalized quantities of the equilibrium theory, which can be written as

$$
Z_{\omega} \sqrt{Z_{\phi} Z_{\tilde{\phi}}^{-1}} \equiv 1
$$

and which implies the following relation between the critical exponents [13]:

$$
z=2+(\tilde{\eta}-\eta) / 2 .
$$

In the nonequilibrium case considered here, such a constraint does not exist and four truly independent critical exponents are present in the theory as compared with three in the $O(2)$ dynamic model A.

\section{E. Results to one-loop order}

To one-loop order in perturbation theory, the $Z$ factors, as well as the phase factor $\theta$ and the parameter $c_{a}$, are not renormalized. We define the following reduced parameters:

$$
\begin{gathered}
\bar{r}=r / c \Lambda^{2}, \quad \bar{\omega}_{0}=\omega_{0} / c \Lambda^{2}, \quad \bar{c}_{a}=c_{a} / c, \\
\bar{u}=u /\left(c \Lambda^{2}\right)^{2}, \quad \bar{u}_{a}=u_{a} /\left(c \Lambda^{2}\right)^{2}, \\
\mathcal{D}=\frac{4 D \Lambda^{d}}{(4 \pi)^{d / 2} \Gamma(d / 2)},
\end{gathered}
$$

to express the RG flow in $d=4-\epsilon$. It is given by three coupled equations

$$
\begin{gathered}
\frac{d \bar{r}}{d l}=2 \bar{r}+2 \mathcal{D} \frac{\bar{u}}{1+\bar{r}}, \\
\frac{d \bar{u}}{d l}=\epsilon \bar{u}-\mathcal{D}\left[\frac{\left(\bar{u}^{2}-\bar{u}_{a}^{2}\right)(1+\bar{r})+2 \bar{u} \bar{u}_{a} \bar{c}_{a}}{(1+\bar{r})\left[\bar{c}_{a}^{2}+(1+\bar{r})^{2}\right]}+\frac{4 \bar{u}^{2}}{(1+\bar{r})^{2}}\right], \\
\frac{d \bar{u}_{a}}{d l}=\epsilon \bar{u}_{a}+\mathcal{D} \frac{\bar{c}_{a}}{1+\bar{r}}\left[\frac{\left(\bar{u}^{2}-\bar{u}_{a}^{2}\right)(1+\bar{r})+2 \bar{u} \bar{u}_{a} \bar{c}_{a}}{(1+\bar{r})\left[\bar{c}_{a}^{2}+(1+\bar{r})^{2}\right]}\right] \\
-6 \mathcal{D} \frac{\bar{u} \bar{u}_{a}}{(1+\bar{r})^{2}},
\end{gathered}
$$

and a fourth one associated with the renormalization of the oscillation frequency,

$$
\frac{d \bar{\omega}_{0}}{d l}=2 \mathcal{D} \frac{\bar{u}_{a}}{1+\bar{r}} e^{-2 l} .
$$

This last equation has to be integrated after the system (53) has been solved.

Since the coefficient $\bar{c}_{a}$ is not renormalized to first order, we find one infrared stable fixed point for each of its values. It is given by

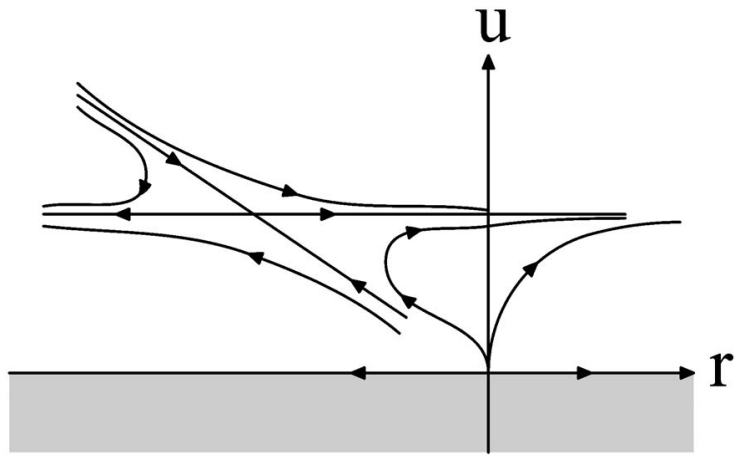

FIG. 1. Qualitative representation of RG flow to one-loop order in perturbation theory, projected on the plane $(r, u)$ and for a space dimension $d<4$.

$$
\bar{r}^{*}=-\frac{\epsilon}{5}, \quad \bar{u}^{*}=\frac{\epsilon}{5 \mathcal{D}}, \quad \text { and } \quad \bar{u}_{a}^{*}=\bar{c}_{a} \frac{\epsilon}{5 \mathcal{D}} .
$$

Writing $\bar{r}=\bar{r}^{*}+\delta r, \bar{u}=\bar{u}^{*}+\delta u$, and $\bar{u}_{a}=\bar{u}_{a}^{*}+\delta u_{a}$, the linearized $\mathrm{RG}$ equations at the fixed point are given by

$$
\frac{d}{d l}\left(\begin{array}{l}
\delta r \\
\delta u \\
\delta u_{a}
\end{array}\right)=\left(\begin{array}{ccc}
2(1-\epsilon / 5) & 2 \mathcal{D} & 0 \\
0 & -\epsilon & 0 \\
0 & -4 \bar{c}_{a} \epsilon / 5 & -\epsilon / 5
\end{array}\right)\left(\begin{array}{c}
\delta r \\
\delta u \\
\delta u_{a}
\end{array}\right) .
$$

The RG flow of the theory is three-dimensional. We show in Fig. 1 the qualitative RG flow projected on the plane $(r, u)$. Finally, the critical exponents to the one-loop order read

$$
\nu=\frac{1}{2}+\frac{\epsilon}{10}, \quad z=2, \quad \eta=0, \quad \tilde{\eta}=0 .
$$

To one-loop order, the three $Z$ factors and the parameter $c_{a}$ are not renormalized. Therefore, these calculations are insufficient to determine the fixed-point value of $c_{a}$ as well as three of the four independent critical exponents of the theory. Calculations to two-loop order are necessary to obtain the full RG structure and the critical properties. Wilson's momentum shell RG scheme is not well suited to perform such calculations and it is technically far more convenient to perform these using a Callan-Symanzik RG scheme, adapted to the renormalization of critical oscillators. This is discussed in the next section.

\section{CALLAN-SYMANZIK RG SCHEME}

The Callan-Symanzik RG scheme avoids the introduction of a cutoff in the momentum space, which is responsible for making the evaluation of multiple integrals technically difficult in Wilson's scheme. In its absence, the calculation of two-loop and higher-order Feynman integrals is easier. Here we present the general structure of Callan-Symanzik's RG scheme adapted to the study of coupled oscillators. We discuss the RG flow to two-loop order in perturbation theory and show that the Callan-Symanzik RG scheme described here is consistent with the momentum shell procedure presented before. 


\section{A. General formalism}

Within a Callan-Symanzik RG scheme [2,13], we start from a bare theory that follows a dynamics of the form (14), whose parameters are labeled with a superscript " 0 ." We then define the renormalized theory such that its effective action is of the form (17). This requires us to introduce a phase shift $\delta \theta$ and a frequency shift $\delta \omega_{0}$ between the bare fields $\left(\phi_{\alpha}^{0}, \widetilde{\phi}_{\alpha}^{0}\right)$ and the renormalized fields $\left(\phi_{\alpha}, \widetilde{\phi}_{\alpha}\right)$, such that

$$
\begin{gathered}
\phi_{\alpha}^{0}\left(\mathbf{x}, t^{0}\right)=\Omega_{\alpha \beta}\left(-\delta \omega_{0} t\right) Z_{\phi}^{1 / 2} Z_{\omega} \phi_{\beta}(\mathbf{x}, t), \\
\widetilde{\phi}_{\alpha}^{0}\left(\mathbf{x}, t^{0}\right)=\Omega_{\alpha \beta}\left(-\delta \theta-\delta \omega_{0} t\right) Z_{\tilde{\phi}}^{1 / 2} Z_{\omega} \widetilde{\phi}_{\beta}(\mathbf{x}, t) .
\end{gathered}
$$

Here we have introduced $Z$ factors for the renormalization of the fields and the time $\left(t^{0}=Z_{\omega}^{-1} t\right)$. We furthermore introduce dimensionless coupling constants $g$ and $g_{a}$ and a scale parameter $\mu$ with $u=\mu^{\epsilon}(4 \pi)^{-\epsilon / 2} g$ and $u_{a}=\mu^{\epsilon}(4 \pi)^{-\epsilon / 2} g_{a}$. Depending on $\mu$, we relate the bare quantities to the renormalized ones by additional $Z$ factors: $g^{0}=Z_{g}\left(Z_{\tilde{\phi}} Z_{\phi}^{3}\right)^{-1 / 2} g, g_{a}^{0}$ $=Z_{g_{a}}\left(Z_{\tilde{\phi}} Z_{\phi}^{3}\right)^{-1 / 2} g_{a}, r^{0}=r_{c}^{0}+Z_{r} r$, and $c_{a}^{0}=Z_{c_{a}} c_{a}$. The dependence of the renormalized parameters $g, g_{a}$, and $c_{a}$ on $\mu$ defines three $\beta$ functions. Denoting $\vec{g}=\left(g, g_{a}, c_{a}\right)$, we write $\vec{\beta}(\vec{g}, \epsilon)=\mu\left(\partial_{\mu} \vec{g}\right)_{0}$, where $\vec{\beta}=\left(\beta, \beta_{a}, \beta_{c}\right)$ and $\left(\partial_{\mu}\right)_{0}$ denotes differentiation with fixed $u^{0}, u_{a}^{0}$, and $c_{a}^{0}$. Note again that $c$ and $D$ are kept constant under renormalization and that we choose units such that $c=1$ in the following. The renormalized correlation and response functions $G_{\alpha \beta}=\left\langle\phi_{\alpha} \phi_{\beta}\right\rangle_{c}$ and $\gamma_{\alpha \beta}$ $=\left\langle\phi_{\alpha} \widetilde{\phi}_{\beta}\right\rangle_{c}$ are related to the physical observables $C_{\alpha \beta}$ and $\chi_{\alpha \beta}$ via

$$
\begin{gathered}
C_{\alpha \beta}\left(\mathbf{x}, t^{0}\right)=\Omega_{\alpha \sigma}\left(-\omega_{0} t\right) Z_{\phi} Z_{\omega}^{2} G_{\sigma \beta}(\mathbf{x}, t) \\
\chi_{\alpha \beta}\left(\mathbf{x}, t^{0}\right)=\Lambda^{-1} \Omega_{\alpha \sigma}\left(\theta-\omega_{0} t\right)\left(Z_{\phi} Z_{\tilde{\phi}}\right)^{1 / 2} Z_{\omega}^{2} \gamma_{\sigma \beta}(\mathbf{x}, t) .
\end{gathered}
$$

The frequency $\omega_{0}=Z_{\omega}^{-1} \omega_{0}^{0}+\delta \omega_{0}$ and the phase $\theta=\theta^{0}+\delta \theta$ are renormalized according to

$$
\begin{gathered}
\mu\left(\partial_{\mu} \delta \theta\right)_{0}=\gamma_{\theta}\left(g, g_{a}, c_{a}, \epsilon\right), \\
\mu\left(\partial_{\mu} \delta \omega_{0}\right)_{0}=r \gamma_{\omega_{0}}\left(g, g_{a}, c_{a}, \epsilon\right),
\end{gathered}
$$

which defines the functions $\gamma_{\theta}$ and $\gamma_{\omega_{0}}$, which are generalized Wilson's functions. In addition, we define Wilson's functions associated with the dependence of the $Z$ factors on $\mu: \gamma_{r}=\mu\left(\partial_{\mu} \ln Z_{r}\right)_{0}, \quad \gamma_{\omega}=\mu\left(\partial_{\mu} \ln Z_{\omega}\right)_{0}, \quad \gamma=\mu\left(\partial_{\mu} \ln Z_{\phi}\right)_{0}$, and $\tilde{\gamma}=\mu\left(\partial_{\mu} \ln Z_{\tilde{\phi}}\right)_{0}$.

The independence of the bare theory with respect to the scale parameter $\mu$ leads to the Callan-Symanzik equations, which we write in terms of the renormalized theory:

$$
\begin{gathered}
{\left[\frac{\partial}{\partial \ln \mu}+\vec{\beta} \cdot \frac{\partial}{\partial \vec{g}}-\sum_{i(j)=1}^{\tilde{N}(N)} \gamma_{\omega} \frac{\partial}{\partial \ln \omega_{i(j)}}-\gamma_{r} \frac{\partial}{\partial \ln r}\right] \Gamma^{(\tilde{N}, N)}} \\
=\left[(\tilde{N}+N-1) \gamma_{\omega}+\frac{\tilde{N}}{2} \tilde{\gamma}+\frac{N}{2} \gamma\right] \Gamma^{(\tilde{N}, N)} .
\end{gathered}
$$

Here $\Gamma^{(\tilde{N}, N)}$ is the vertex function with $\tilde{N}$ and $N$ truncated external legs corresponding to the fields $\widetilde{\phi}$ and $\phi$, respectively. It is a function of $\tilde{N}$ variables $\left(\omega_{i}, \mathbf{q}_{\mathbf{i}}\right)$ and $N$ variables $\left(\omega_{j}, \mathbf{q}_{\mathbf{j}}\right)$ describing the frequencies and wavelengths associated with all external legs, and depends on the renormalized set of parameters.

In order to calculate Wilson's beta and gamma functions which appear in the Callan-Symanzik equations (61), we decompose the bare action associated with the fields $\phi_{\alpha}^{0}$ and $\widetilde{\phi}_{\alpha}^{0}$ as

$$
\mathcal{S}^{0}\left[\widetilde{\phi}_{\alpha}^{0}, \phi_{\alpha}^{0}\right]=\mathcal{S}_{\mathrm{R}}\left[\widetilde{\phi}_{\alpha}, \phi_{\alpha}\right]+\delta \mathcal{S}\left[\widetilde{\phi}_{\alpha}, \phi_{\alpha}\right]+\mathcal{S}_{\mathrm{Mop}}\left[\widetilde{\phi}_{\alpha}, \phi_{\alpha}\right]
$$

Here $\mathcal{S}_{\mathrm{R}}\left[\widetilde{\phi}_{\alpha}, \phi_{\alpha}\right]$ represents the action of the renormalized theory, $\mathcal{S}_{\text {Mop }}\left[\widetilde{\phi}_{\alpha}, \phi_{\alpha}\right]$ the action associated with the "mass operator" $\left[\widetilde{\phi}_{\alpha} \phi_{\beta}\right]$, and $\delta \mathcal{S}\left[\widetilde{\phi}_{\alpha}, \phi_{\alpha}\right]$ combines the counterterms. The integrals corresponding to the Feynman diagrams of the theory contain poles as a function of the small dimensional parameter $\epsilon$. The $Z$ factors are determined such that the counterterms absorb these poles and the effective action is finite. We write

$$
\begin{aligned}
\mathcal{S}_{\mathrm{R}}\left[\widetilde{\phi}_{\alpha}, \phi_{\alpha}\right]= & \int_{\mathbf{q}, \omega}\left\{D \widetilde{\phi}_{\alpha} \widetilde{\phi}_{\alpha}-\widetilde{\phi}_{\alpha}\left[i \omega+c \mathbf{q}^{2}\right] \phi_{\alpha}\right. \\
& \left.-\widetilde{\phi}_{\alpha}\left[c_{a} \mathbf{q}^{2}\right] \varepsilon_{\alpha \beta} \phi_{\beta}\right\} \\
& -\int_{k_{1} k_{2} k_{3}} \mu^{\epsilon}(4 \pi)^{-\epsilon / 2} g_{\alpha \beta} \widetilde{\phi}_{\alpha} \phi_{\beta} \phi_{\gamma} \phi_{\gamma},
\end{aligned}
$$

$$
\begin{aligned}
\delta \mathcal{S}\left[\widetilde{\phi}_{\alpha}, \phi_{\alpha}\right]= & \int_{\mathbf{q}, \omega}\left\{D\left(Z_{\omega} Z_{\tilde{\phi}}-1\right) \widetilde{\phi}_{\alpha} \widetilde{\phi}_{\alpha}-\widetilde{\phi}_{\alpha}\left[i \omega\left(Z_{\omega}^{2} \sqrt{Z_{\phi} Z_{\tilde{\phi}}} \cos \delta \theta-1\right)+c \mathbf{q}^{2}\left(Z_{\omega} \sqrt{Z_{\phi} Z_{\tilde{\phi}}} \cos \delta \theta-Z_{\omega} \sqrt{Z_{\phi} Z_{\tilde{\phi}}} Z_{c_{a}} c_{a} / c \sin \delta \theta-1\right)\right] \phi_{\alpha}\right. \\
& \left.-\widetilde{\phi}_{\alpha}\left[i \omega\left(Z_{\omega}^{2} \sqrt{Z_{\phi} Z_{\tilde{\phi}}} \sin \delta \theta\right)+c_{a} \mathbf{q}^{2}\left(Z_{\omega} \sqrt{Z_{\phi} Z_{\tilde{\phi}}} Z_{c_{a}} \cos \delta \theta+Z_{\omega} \sqrt{Z_{\phi} Z_{\tilde{\phi}}} c / c_{a} \sin \delta \theta-1\right)\right] \varepsilon_{\alpha \beta} \phi_{\beta}\right\} \\
& -\mu^{\epsilon}(4 \pi)^{-\epsilon / 2} \int_{k_{1} k_{2} k_{3}}\left\{\left[\left(Z_{g} Z_{\omega}^{3} \cos \delta \theta-1\right) g-Z_{g_{a}} Z_{\omega}^{3} g_{a} \sin \delta \theta\right] \widetilde{\phi}_{\alpha} \phi_{\alpha} \phi_{\gamma} \phi_{\gamma}\right. \\
& \left.+\left[\left(Z_{g_{a}} Z_{\omega}^{3} \cos \delta \theta-1\right) g_{a}+Z_{g} Z_{\omega}^{3} g \sin \delta \theta\right] \varepsilon_{\alpha \beta} \widetilde{\phi}_{\alpha} \phi_{\beta} \phi_{\gamma} \phi_{\gamma}\right\}
\end{aligned}
$$




$$
\begin{aligned}
& \mathcal{S}_{\text {Mop }}\left[\widetilde{\phi}_{\alpha}, \phi_{\alpha}\right] \\
& \quad=\int_{\mathbf{q}, \omega}\left\{-\widetilde{\phi}_{\alpha} \phi_{\alpha} \sqrt{Z_{\phi} Z_{\tilde{\phi}}} Z_{\omega}\left(r Z_{r} \cos \delta \theta\right)\right\} \\
& \quad+\int_{\mathbf{q}, \omega}\left\{-\widetilde{\phi}_{\alpha} \varepsilon_{\alpha \beta} \phi_{\beta} \sqrt{Z_{\phi} Z_{\tilde{\phi}}} Z_{\omega}\left[-Z_{\omega} \delta \omega_{0}+r Z_{r} \sin \delta \theta\right]\right\},
\end{aligned}
$$

where

$$
g_{\alpha \beta}=\left(\begin{array}{cc}
g & -g_{a} \\
g_{a} & g
\end{array}\right) .
$$

Having determined the $Z$ factors, we can calculate the $\beta$ functions. Writing

$$
\begin{gathered}
\beta\left(g, g_{a}, c_{a}, \epsilon\right)=-\epsilon g+\beta^{(4)}\left(g, g_{a}, c_{a}\right), \\
\beta_{a}\left(g, g_{a}, c_{a}, \epsilon\right)=-\epsilon g_{a}+\beta_{a}^{(4)}\left(g, g_{a}, c_{a}\right), \\
\beta_{c}\left(g, g_{a}, c_{a}, \epsilon\right)=\beta_{c}^{(4)}\left(g, g_{a}, c_{a}\right),
\end{gathered}
$$

the functions $\beta^{(4)}$ do not depend on $\epsilon$ and are given by

$$
\left(\begin{array}{c}
\beta^{(4)} \\
\beta_{a}^{(4)} \\
\beta_{c}^{(4)}
\end{array}\right)=\left(\begin{array}{ccc}
g \frac{\partial \bar{Z}_{g}}{\partial g} & g \frac{\partial \bar{Z}_{g}}{\partial g_{a}} & g \frac{\partial \bar{Z}_{g}}{\partial c_{a}} \\
g_{a} \frac{\partial \bar{Z}_{g_{a}}}{\partial g} & g_{a} \frac{\partial \bar{Z}_{g_{a}}}{\partial g_{a}} & g_{a} \frac{\partial \bar{Z}_{g_{a}}}{\partial c_{a}} \\
c_{a} \frac{\partial Z_{c_{a}}}{\partial g} & c_{a} \frac{\partial Z_{c_{a}}}{\partial g_{a}} & Z_{c_{a}}+c_{a} \frac{\partial Z_{c_{a}}}{\partial c_{a}}
\end{array}\right)\left(\begin{array}{c}
g \\
g_{a} \\
0
\end{array}\right),
$$

where the superscript "(1)" indicates the coefficients of the poles in $\epsilon^{-1}$ of the different $Z$ factors in the matrix. Here $\bar{Z}_{g}=Z_{g}\left(Z_{\tilde{\phi}} Z_{\phi}^{3}\right)^{-1 / 2}$ and $\bar{Z}_{g_{a}}=Z_{g_{a}}\left(Z_{\tilde{\phi}} Z_{\phi}^{3}\right)^{-1 / 2}$. Wilson's gamma functions of the theory are independent of $\epsilon$. We have

$$
\gamma_{r}\left(g, g_{a}, c_{a}\right)=-g \frac{\partial Z_{r}^{(1)}}{\partial g}-g_{a} \frac{\partial Z_{r}^{(1)}}{\partial g_{a}},
$$

and corresponding expressions for $\gamma_{\omega}, \gamma$, and $\tilde{\gamma}$. Furthermore,

$$
\begin{gathered}
\gamma_{\theta}\left(g, g_{a}, c_{a}\right)=-g \frac{\partial \delta \theta^{(1)}}{\partial g}-g_{a} \frac{\partial \delta \theta^{(1)}}{\partial g_{a}}, \\
\gamma_{\omega_{0}}\left(g, g_{a}, c_{a}\right)=\frac{1}{r}\left(-g \frac{\partial \delta \omega_{0}^{(1)}}{\partial g}-g_{a} \frac{\partial \delta \omega_{0}^{(1)}}{\partial g_{a}}\right) .
\end{gathered}
$$

The RG fixed points correspond to the values $\vec{g}^{*}$ of $\vec{g}$ for which the $\beta$ functions are zero. The critical exponents of the theory are given by the fixed-point values of the gamma functions. We have

$$
\begin{gathered}
\nu=\left[2+\gamma_{r}\left(\vec{g}^{*}\right)\right]^{-1}, \quad z=2+\gamma_{\omega}\left(\vec{g}^{*}\right), \\
\eta=\gamma\left(\vec{g}^{*}\right)+2 \gamma_{\omega}\left(\vec{g}^{*}\right), \quad \tilde{\eta}=\tilde{\gamma}\left(\vec{g}^{*}\right)+2 \gamma_{\omega}\left(\vec{g}^{*}\right) .
\end{gathered}
$$

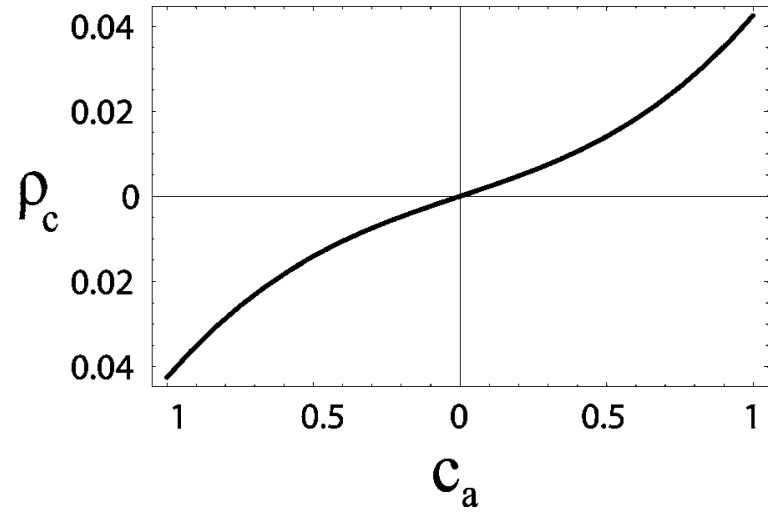

FIG. 2. The renormalization of the parameter $c_{a}$ is described by the Wilson function $\rho_{c}$, whose expression to two-loop order is given by Eq. (C2) and which is displayed here as a function of $c_{a}$. A fixed point of the theory is characterized by $\rho_{c}=0$. A single fixed point exists for $c_{a}^{*}=0$.

\section{B. One-loop order calculations}

As in the framework of Wilson's momentum shell integration scheme, to one-loop order in perturbation theory, only the parameters $\omega_{0}, r, g$, and $g_{a}$ are renormalized. We find $\gamma_{r}=-2 \mathcal{D} g$ and $\gamma_{\omega_{0}}=2 \mathcal{D} g_{a}$, where $\mathcal{D}=4 D /(4 \pi)^{2}$ (see Appendix B for details about the calculations). Furthermore, the $\beta$ functions associated with the renormalization of $g$ and $g_{a}$ read

$$
\beta \simeq-\epsilon g-\mathcal{D}\left[\frac{g_{a}^{2}-g^{2}-2 g g_{a} c_{a}}{1+c_{a}^{2}}-4 g^{2}\right],
$$

$$
\beta_{a} \simeq-\epsilon g_{a}-\mathcal{D}\left[\frac{c_{a}}{1+c_{a}^{2}}\left(g^{2}-g_{a}^{2}+2 g g_{a} c_{a}\right)-6 g g_{a}\right] .
$$

We find the same results as those discussed in Sec. V. Choosing $c_{a}$ as a parameter, the fixed points read

$$
g^{*} \simeq \epsilon / 5 \mathcal{D}, \quad g_{a}^{*} \simeq c_{a} \epsilon / 5 \mathcal{D} .
$$

\section{Two-loop order calculations and universality class}

To this order in perturbation theory, all $Z$ factors and parameters of the theory are renormalized. Details about this renormalization procedure are given in the appendixes: Appendix A gives graphic representations of the Feynman diagrams of the perturbation theory, Appendix B gives the main calculation steps and explicit expressions for the associated integrals, and Appendix $\mathrm{C}$ provides expressions for the different Wilson functions of the theory, including $\beta_{c}\left(g, g_{a}, c_{a}\right)$. The renormalization of the propagator to second order does not affect the first-order results discussed above. The fixedpoint condition for the full problem can thus be written in the form $\rho_{c}\left(c_{a}^{*}\right)=0$, where 


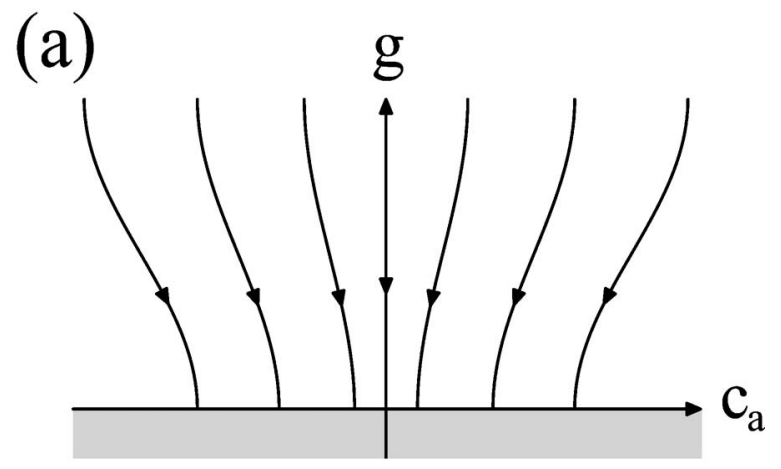

(b)

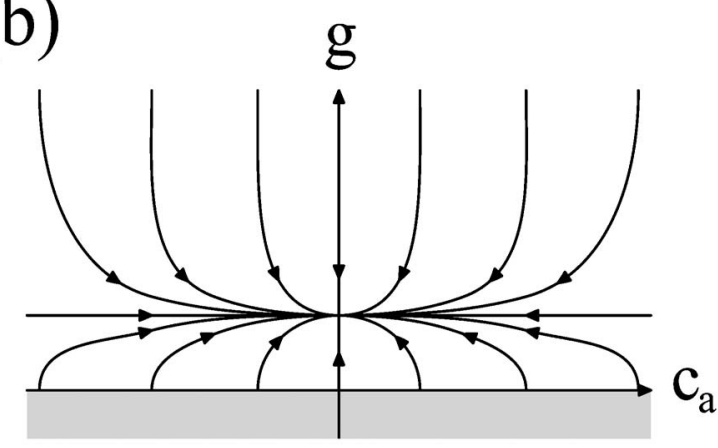

FIG. 3. Schematic representation of the RG flow of the theory, obtained to two-loop order in perturbation theory, and projected on the plane $\left(g, c_{a}\right)$. (a) Space dimension $d>4$. (b) Space dimension $d<4$.

$$
\rho_{c}\left(c_{a}\right)=\epsilon^{-2} \beta_{c}\left(g^{*}, g_{a}^{*}, c_{a}\right)
$$

and $c_{a}^{*}$ denotes the fixed-point value of $c_{a}$. Here, we used the fixed-point values $g^{*}$ and $g_{a}^{*}$ given by Eq. (73). Note that to two-loop order in perturbation theory, this function $\rho_{c}\left(c_{a}\right)$ is independent of $\epsilon$. The full expression of the function $\rho_{c}\left(c_{a}\right)$ is given in Appendix C, Eq. (C2), and its graphic representation in Fig. 2.

A single fixed point of the theory exists with $c_{a}^{*}=0$ and $g_{a}^{*}=0$. This fixed point is IR-stable. It is the same as the one of the real Ginzburg-Landau theory with $O(2)$ symmetry. As a consequence, the dominant critical exponents are the same as those known for the $O(2)$ dynamic model A. They are $\nu$ $\simeq 1 / 2+\epsilon / 10, \quad \eta \simeq \epsilon^{2} / 50$, and $z \simeq 2+\epsilon^{2}[6 \ln (4 / 3)-1] / 50$, associated with the relation $z=2+(\tilde{\eta}-\eta) / 2$. Furthermore, at this fixed point, both functions $\gamma_{\theta}$ and $\gamma_{\omega_{0}}$ are equal to zero. Therefore, at the critical point, the effective phase and frequency become scale-invariant. Note, however, that since the renormalized fields differ from the physical fields by time-dependent variable transformations, the physical correlation and response functions differ significantly from those associated with the $O(2)$ dynamic model. The asymptotic expressions of these functions are discussed is Sec. VII.

The critical behaviors of the theory are characterized by the linearized RG flow in the vicinity of the fixed point. The linearized flow equations can be written as

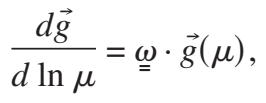

where we have introduced the matrix

$$
\stackrel{\omega}{=}=\left(\begin{array}{ccc}
\epsilon & 0 & 0 \\
0 & \epsilon / 5 & -\epsilon^{2} / 25 \mathcal{D} \\
0 & 0 & \epsilon^{2} / 50
\end{array}\right) .
$$

The eigenvalues of this matrix are

$$
\omega_{1}=\varepsilon, \quad \omega_{2}=\frac{\varepsilon}{5}, \quad \omega_{3}=\frac{\varepsilon^{2}}{50} .
$$

In addition to $\omega_{1}$, which is known from the $O(2)$ symmetric dynamic model $\mathrm{A}$, we find here two new universal quantities $\omega_{2}$ and $\omega_{3}$, which are specific to critical oscillators.

\section{Flow diagram of the theory to two-loop order in perturbation theory}

The RG flow of the theory within the Callan-Symanzik $\mathrm{RG}$ scheme is given by the variations of the three parameters $g, g_{a}$, and $c_{a}$ under renormalization. We display in Fig. 3 the projection of this flow on the plane $\left(g, c_{a}\right)$ for two different space dimensions, above and below the upper critical dimension $d_{c}=4$. The first plot corresponds to $d>4$ and the second to $d<4$.

Because the RG flow here is defined in an enlarged space, its structure differs remarkably from that of the real Ginzburg-Landau theory. For $d>4$, we find a line of Gaussian fixed points corresponding to $g^{*}=0$ and $g_{a}^{*}=0$ for any value of $c_{a}^{*}$. These fixed points characterize the mean-field universality classes of critical oscillators, which depend on the value of $c_{a}^{*}$. Below the critical dimension $d_{c}=4$, a single fixed point exists with $g^{*}=\epsilon / 5 \mathcal{D}, g_{a}^{*}=0$, and $c_{a}^{*}=0$. Because of the existence of a whole line of Gaussian fixed points that change their stability at $d=d_{c}$, the RG flow has a singular structure near $d=d_{c}$. As a consequence, the large-scale behaviors of critical oscillators for $d>d_{c}$ can vary correspondingly to different values of the effective parameter $c_{a}$. For $d<d_{c}$, however, the characteristic critical behaviors are always described by the single fixed point with $c_{a}^{*}=0$, relevant for this case.

\section{CORRELATION AND RESPONSE FUNCTIONS AND VIOLATION OF THE FLUCTUATION-DISSIPATION RELATION}

In the previous section, we discussed the RG flow and fixed-point structures of the theory. We saw that the renormalized fields are described in a reference frame that oscillates with renormalized frequency and phase factors. The fixed-point theory is formally equivalent to that of a critical point at thermodynamic equilibrium, namely the critical point of the dynamic Ginzburg-Landau theory with an $O(2)$ symmetry. However, we show now that the correlation and response functions of the physical fields studied here have different properties. 


\section{A. Asymptotic behaviors of the correlation and response functions in the critical regime}

The asymptotic behaviors of the correlation and response functions of the theory near criticality can be determined using the RG flow and applying a matching procedure to link these functions with their expressions off criticality (see, e.g., [38]). In the present case, such a matching procedure needs to be adapted. Indeed, the physical correlation and response functions are related to those defined for the renormalized fields by the time-dependent transformations (59). We therefore have to add to the usual matching procedure a scale-dependent transformation to describe the physical theory in its original reference frame. This transformation depends on the effective frequency and phase of the oscillators, which are renormalized by the RG procedure. Taking all this into account, we can write effective asymptotic expressions for the functions associated with physical quantities.

The effective linear-response function of the physical theory behaves as (for $q \xi \gg 1$ and for stimulation at the effective frequency $\omega_{0}^{\text {eff }}$ )

$$
\chi\left(q, \omega=\omega_{0}^{\mathrm{eff}}\right) \simeq \frac{1}{q^{2-\eta}} \frac{1}{2 \Lambda_{\mathrm{eff}}}\left[\frac{e^{i \theta(q)}}{c_{\mathrm{eff}}+i \gamma(q)}\right] .
$$

Here, we denote by $\omega_{0}^{\text {eff }}$ the effective oscillation frequency at the bifurcation, and by $\xi$ the correlation length in the nonoscillating phase. Furthermore, we have introduced the functions $\theta(q) \simeq \theta_{\mathrm{eff}}+\alpha_{\mathrm{eff}} q^{\omega_{2}}+\beta_{\mathrm{eff}} q^{\omega_{3}}$ and $\gamma(q) \simeq \gamma_{\mathrm{eff}} q^{\omega_{3}}$ of the wave number $q=|\mathbf{q}|$, as well as nonuniversal effective quantities denoted by the index "eff." These functions are derived, respectively, from the renormalizations of the parameters $\theta$ and $c_{a}$ in the vicinity of the fixed point. Note that they depend on the universal critical exponents given by Eq. (77). Similarly to Eq. (78), the correlation function behaves as

$$
C\left(q, \omega=\omega_{0}^{\mathrm{eff}}\right) \simeq \frac{1}{q^{z+2-\eta}} \frac{D_{\mathrm{eff}}}{c_{\mathrm{eff}}^{2}+\gamma(q)^{2}} .
$$

Related expressions can be obtained for the frequency dependence for $q=0$ in the regime $\left(\omega-\omega_{0}^{\text {eff }}\right) \xi^{z} \gg 1$. They are given by

$$
\begin{gathered}
\chi(q=0, \omega) \simeq \pm i \frac{e^{i \theta\left(\omega-\omega_{0}^{\mathrm{eff}}\right)}}{2 \Lambda_{\mathrm{eff}}} \frac{1}{\left|\omega-\omega_{0}^{\mathrm{eff}}\right|(2-\eta) / z[\cdot]^{(2-\eta) / z}}, \\
C(q=0, \omega) \simeq \frac{D_{\mathrm{eff}}}{\left|\omega-\omega_{0}^{\mathrm{eff}}\right|(2+z-\eta) / z[\cdot]^{(2+z-\eta) / z}},
\end{gathered}
$$

where

$$
\begin{gathered}
{[\cdot] \simeq\left[1+\rho_{\text {eff }}\left|\omega-\omega_{0}^{\text {eff }}\right| \omega_{2} / 2+\sigma_{\text {eff }}\left|\omega-\omega_{0}^{\text {eff }}\right|(1 / 2 \nu)-1\right],} \\
\theta\left(\omega-\omega_{0}^{\text {eff }}\right) \simeq \theta_{\text {eff }}+\alpha_{\text {eff }}\left|\omega-\omega_{0}^{\text {eff }}\right| \omega_{2} / z+\beta_{\text {eff }}\left|\omega-\omega_{0}^{\text {eff }}\right| \omega_{3} / z
\end{gathered}
$$

and where " \pm " corresponds to $\omega-\omega_{0}^{\text {eff }}$ being positive or negative, respectively. The anomalous dependences on frequencies as given by Eq. (81) are due to the nontrivial evolutions of the parameters $\theta$ and $\omega_{0}$ under renormalization.

\section{B. Generalized fluctuation-dissipation relation in the critical regime}

A collective system close to a Hopf bifurcation operates far from thermodynamic equilibrium. Therefore, the correlation function $C_{\alpha \beta}$ and the linear-response function $\chi_{\alpha \beta}$ do not obey the fluctuation-dissipation (FD) relation that is characteristic of thermodynamic equilibrium. Interestingly, the effective theory at the RG fixed point, expressed in terms of the renormalized fields $\phi_{\alpha}$ and $\widetilde{\phi}_{\alpha}$, is formally equivalent to a fixed-point theory at thermodynamic equilibrium. Therefore, exactly at the fixed point, a relation appears between the correlation and response functions $G_{\alpha \beta}$ and $\gamma_{\alpha \beta}$ of the renormalized fields. It takes the form

$$
G_{\alpha \beta}=\frac{2 D}{\omega} \gamma_{\alpha \beta}^{\prime \prime}
$$

where $\gamma_{\alpha \beta}=\gamma_{\alpha \beta}^{\prime}+i \gamma_{\alpha \beta}^{\prime \prime}$ has been split into its real and imaginary parts. The emergence of this relation in the critical regime can be discussed by the introduction of the function [53]

$$
F(\mathbf{q}, \omega)=\frac{2 D}{\omega} \frac{\gamma_{11}^{\prime \prime}(\mathbf{q}, \omega)}{G_{11}(\mathbf{q}, \omega)} .
$$

The evolution of this quantity under renormalization is described by the following Callan-Symanzik equation:

$$
\begin{gathered}
{\left[\frac{\partial}{\partial \ln \mu}+\vec{\beta} \cdot \frac{\partial}{\partial \vec{g}}-\gamma_{\omega} \frac{\partial}{\partial \ln \omega}-\gamma_{r} \frac{\partial}{\partial \ln r}\right] F} \\
=\left[\gamma_{\omega}+\frac{1}{2}(\gamma-\tilde{\gamma})\right] F .
\end{gathered}
$$

Since the fixed-point theory obeys the FD relation, we have $F\left(\mathbf{q}, \omega, r, \vec{g}^{*}, \mu\right)=1$. Note that in mean-field theory we find $F^{\mathrm{mf}}=\left[\omega^{2}+R^{2}-\left(c_{a} q^{2}\right)^{2}\right] /\left[\omega^{2}+R^{2}+\left(c_{a} q^{2}\right)^{2}\right]$, which differs from $F=1$ if $c_{a} \neq 0$.

The fact that the renormalized theory at the fixed point obeys the FD relation $F=1$ implies that the correlation and response functions $C_{\alpha \beta}$ and $\chi_{\alpha \beta}$ at that point are not independent. Since they are related to $G_{\alpha \beta}$ and $\gamma_{\alpha \beta}$ by Eq. (59), we find

$$
\begin{aligned}
& \cos \theta_{\text {eff }} \chi_{11}^{\prime \prime}+\sin \theta_{\text {eff }} \chi_{12}^{\prime \prime}=\frac{1}{2 \Lambda_{\text {eff }} D_{\text {eff }}}\left(\omega C_{11}+i \omega_{0}^{\text {eff }} C_{12}\right), \\
& \cos \theta_{\text {eff }} \chi_{12}^{\prime}-\sin \theta_{\text {eff }} \chi_{11}^{\prime}=\frac{1}{2 \Lambda_{\text {eff }} D_{\text {eff }}}\left(\omega_{0}^{\text {eff }} C_{11}+i \omega C_{12}\right)
\end{aligned}
$$

Here again $\chi_{\alpha \beta}=\chi_{\alpha \beta}^{\prime}+i \chi_{\alpha \beta}^{\prime \prime}$ has been separated in its real and imaginary parts. At the bifurcation, this relation is asymptotically satisfied in the long-time and wavelength limits. It is a consequence of symmetry properties of the fixed-point theory which impose constraints on the correlation and response functions at criticality. Indeed, the FD relation is connected with time-reversal invariance, which emerges for the fields $\phi_{\alpha}$ and $\widetilde{\phi}_{\alpha}$ at criticality while it is not obeyed for the physical fields $\psi_{\alpha}$ and $\widetilde{\psi}_{\alpha}$. 


\section{Breaking of the fluctuation-dissipation relation}

The relation between the physical correlation and response functions at criticality given by Eq. (85) is not an FD relation. In order to characterize the violation of the FD relation between $C_{\alpha \beta}$ and $\chi_{\alpha \beta}$, we define an effective temperature $T_{\text {eff }}$, which depends on frequency and wave vector [39],

$$
\frac{T_{\mathrm{eff}}(\mathbf{q}, \omega)}{T}=\frac{\omega}{2 k_{B} T} \frac{C_{11}(\mathbf{q}, \omega)}{\chi_{11}^{\prime \prime}(\mathbf{q}, \omega)} .
$$

Here, $k_{B}$ denotes the Boltzmann constant and $T$ is the temperature. Using the previous asymptotic expressions for the two-point correlation and response functions, we find universal behaviors of this effective temperature at criticality,

$$
\begin{gathered}
T_{\mathrm{eff}}\left(q, \omega=\omega_{0}^{\mathrm{eff}}\right) / T \sim q^{-z}, \\
T_{\mathrm{eff}}(q=0, \omega) / T \sim\left|\omega-\omega_{0}^{\mathrm{eff}}\right|^{-\sigma} .
\end{gathered}
$$

For the particular case $c_{a}=0$ and $u_{a}=0, \sigma=1$, while otherwise $\sigma \simeq 1-\epsilon / 5$ to first order in $\epsilon$ [54]. This singular behavior of the effective temperature implies a violent breaking of the FD relation. This is consistent with the fact that spontaneously oscillating systems operate far from thermodynamic equilibrium.

\section{SUMMARY AND CONCLUSION}

We have studied the critical behaviors of a large number of locally coupled oscillators when approaching a homogeneous synchronization transition from the disordered phase in a $d=(4-\epsilon)$-dimensional space. On large length and time scales, the critical behaviors can be described by a statistical field theory that is given by the complex Ginzburg-Landau equation with additional noise and forcing terms. At the critical point of a homogeneous oscillatory instability, timetranslational invariance is spontaneously broken in the system. The field variable $Z$ in the complex Ginzburg-Landau field theory is constructed in such a way that time translations correspond to global phase changes of this complex variable $Z$. Within this framework, breaking of timetranslational invariance becomes formally similar to the traditional spontaneous symmetry breaking known for other second-order phase transitions.

We have established the structure of the associated dynamic RG within Wilson's and Callan-Symanzik's schemes, and performed the calculations to two-loop order in perturbation theory. We have shown that the critical point is formally related to the equilibrium phase transition in the real Ginzburg-Landau $O(2)$ dynamic model A. However, the RG flow of critical oscillators is defined in a larger parameter space of nonequilibrium field theories and leads to a renormalization of oscillation frequency and phase. The FD relation is broken in the system, which can be characterized by an effective frequency-dependent temperature, diverging at the effective oscillation frequency with an anomalous power law.

The formal analogy with an $O(2)$ symmetric dynamic field theory, valid at the critical point, leads to several inter- esting results. For $d>2$, the collective dynamics of coupled oscillators exhibits a second-order nonequilibrium phase transition. This phase transition is a generalization of Hopf bifurcations, which are conventionally defined in the context of nonlinear dynamics, to nonequilibrium statistical physics. On the oscillating side of the bifurcation and in the thermodynamic limit, the system exhibits long-range phase order and coherent oscillations. In mean-field theory, the universal properties of this oscillating instability are captured by the normal form known from nonlinear dynamics. Below the upper critical dimension, however, fluctuations become relevant and anomalous scaling laws and critical exponents appear.

The case of critical coupled oscillators studied here provides a further example for the emergence of an equilibrium universality class in a nonequilibrium dynamic field theory. In nonequilibrium systems with a nonconserved order parameter, detailed balance is often effectively restored at criticality [17]. This is the case, e.g., for the model A dynamics of the real Ginzburg-Landau theory with $Z_{2}$ symmetry $[40,41]$, even when the symmetry is broken by the nonequilibrium perturbations [42], and for some of its generalizations to the $O(n)$ symmetry [43]. In the present case, the detailed balance condition is not restored for the physical variables, but appears only in the oscillating reference frame associated with the effective frequency and phase of the oscillations at the transition. This emergence of detailedbalance symmetry at criticality imposes a generic relation between the correlation and response functions of coupled oscillators as given by Eq. (85).

The structure of the RG flow studied here is singular at the upper critical dimension $d_{c}=4$. Indeed, as depicted in Fig. 3, the line of Gaussian fixed points, which is stable above $d=4$, becomes unstable for $d<4$ where only one isolated stable fixed point remains. Our results obtained in an epsilon expansion are valid close to the upper critical dimension for $d=4-\epsilon$. We can speculate how our results are modified in lower dimensions $d$. In analogy with the equilibrium $O(2)$ dynamic model, we expect the phase order of the oscillations to vanish for $d<2$, and to be quasi-long-range exactly at the lower critical dimension $d=2$. In the last case, spectral peaks on the oscillating side of the Hopf bifurcation are expected to exhibit power-law tails with nonuniversal exponents. If the formal analogy with the equilibrium critical point found here in $d=4-\epsilon$ persists in $d=2$, we would expect to see features of the Kosterlitz-Thouless universality class [44] in systems of coupled oscillators in this dimension.

The different values of the space dimension $d$ of coupled oscillators can be related to different realizations of coupled nonlinear oscillators in various physical and biological systems. The mean-field limit $d>4$ is found in systems where oscillators are coupled by long-range interactions. Examples for such a situation are sarcomere oscillations in muscles. There, large numbers of myosin motor proteins generate oscillations when interacting with actin filaments, which represent tracks along which the motor proteins move. Oscillations occur if the motor collection acts against elastic elements and in the presence of a chemical fuel that supplies the necessary energy. For stiff filaments, this 
situation is well described by globally coupled motor proteins for which mean-field theory applies at the Hopf bifurcation [45].

Systems of coupled oscillators in three dimensions could be realized in oscillatory chemical processes in bulk solution. This is the case, e.g., of the Belousov-Zhabotinsky reaction which can be studied in the framework of the complex Ginzburg-Landau equation (see, e.g., [46]). On mesoscopic scales, the system can be viewed as a collection of interacting volume elements, each representing an individual chemical oscillator. There, oscillations are subject to fluctuations due to the finite number of reacting molecules present in each volume element.

Coupled oscillators in two dimensions can be realized by oscillators arranged on a surface. Such a situation may occur in the electrosensory organ of some fish species where many electrically oscillating cells constitute the sensory epithelium $[47,48]$. Critical oscillators coupled in two dimensions can also in principle be realized in artificial systems. Nanotechnology aims to build functional units on the submicrometer scale. Large arrays of nano-oscillators on patterned substrates coupled to their neighbors by elastic or viscous effects would provide a two-dimensional realization of our field theory. Finally, the case $d=0$ corresponds to a single noisy oscillator. Here, fluctuations destroy the Hopf bifurcation and only its signatures can be observed. In the context of biological systems, an example is the spontaneous oscillations of the mechanosensory organelle of auditory hair cells $[39,49]$. Here, the critical divergence of the linear-response function is ideally suited for signal detection.

In order to observe the critical exponents discussed here, homogeneous chemical oscillations in a bulk system with $d$ $=3$ (which corresponds here to $\epsilon=1$ ) would be a good candidate. However, the critical exponents attached to the RG fixed point are only observable when the system is observed sufficiently close to the critical point. The range and experimental accessibility of this critical regime can be estimated by a Ginzburg criterion, see Appendix D. Assuming that in a chemical system the Hopf bifurcation occurs if a molecular concentration $\rho$ exceeds a critical value $\rho_{c}$, the critical regime corresponds to

$$
\frac{\left|\rho-\rho_{c}\right|}{\rho_{c}}<\frac{\omega_{0}}{\rho^{2} \tau_{c}^{2} c^{3}},
$$

where $\omega_{0}$ is the oscillation frequency and $\tau_{c}$ denotes a chemical reaction time. The coefficient $c$ here is the bare coefficient describing the coupling of oscillators in the complex Ginzburg-Landau equation. We can rewrite this expression as

$$
\frac{\left|\rho-\rho_{c}\right|}{\rho_{c}}<\frac{\omega_{0} \tau_{c}}{\left(\rho l_{c}^{3}\right)^{2}}\left(\frac{D_{m}}{c}\right)^{3},
$$

where $l_{c}^{2}=D_{m} \tau_{c}$ is a reaction length and $D_{m}$ denotes a microscopic diffusion coefficient. Since $\omega_{0} \tau_{c} \ll 1$ (oscillations are slow compared to fast reaction times) and $l_{c}^{3} \rho \gg 1$ (the volume per molecule is small compared to the reaction volume), accessibility to the critical regime requires that $D_{m} \gg c$. This condition is satisfies if $c$ becomes small. This happens in
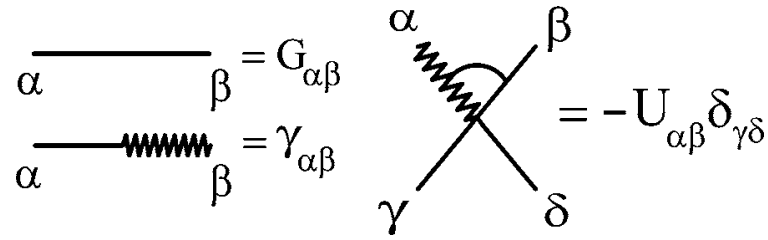

FIG. 4. Graphic representation of the propagators $G_{\alpha \beta}$ and $\gamma_{\alpha \beta}$, and of the vertex $U_{\alpha \beta} \delta_{\gamma \delta}$.

particular if a Turing instability is approached. At the point where such an instability occurs, the coefficient $c$ changes sign and stationary spatial patterns appear. Our analysis suggests that before such a point is reached, the critical regime of the Hopf bifurcation becomes accessible. Therefore, the scaling behaviors and critical exponents discussed here could be experimentally observable in oscillating chemical systems.

\section{ACKNOWLEDGMENTS}

We thank Edouard Brézin, Erwin Frey, and Kay Wiese for useful discussions.

\section{APPENDIX A: FEYNMAN DIAGRAMS OF THE PERTURBATION THEORY}

Here, we present the graphic representation of the terms of the expansion series that we used for the calculations to one- and two-loop orders in perturbation theory. The expansion series of the action of the complex Ginzburg-Landau theory given by Eq. (17) can be represented by Feynman diagrams as usual. The free propagator of the theory, calculated from the Gaussian part of the action $\mathcal{S}_{\mathrm{R}}$ of the decomposition (62) in a Callan-Symanzik RG scheme, is given by

$$
\begin{aligned}
& G_{\alpha \beta}^{0}(\mathbf{q}, \omega) \\
& \quad=\frac{2 D}{|\Delta|^{2}}\left(\begin{array}{cc}
\omega^{2}+\left(c_{a} \mathbf{q}^{2}\right)^{2}+\left(c \mathbf{q}^{2}\right)^{2} & 2 i \omega c_{a} \mathbf{q}^{2} \\
-2 i \omega c_{a} \mathbf{q}^{2} & \omega^{2}+\left(c_{a} \mathbf{q}^{2}\right)^{2}+\left(c \mathbf{q}^{2}\right)^{2}
\end{array}\right),
\end{aligned}
$$

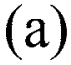

(b)
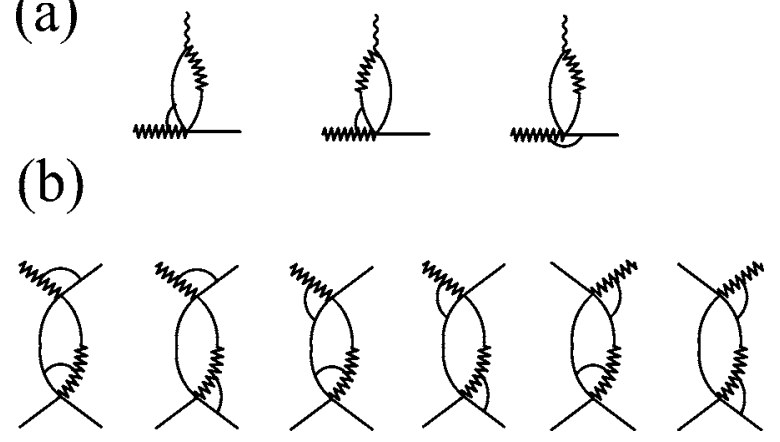

FIG. 5. Feynman diagrams contributing to the renormalization of the theory to one-loop order. (a) Renormalization of the "mass operator" $\left[\widetilde{\phi}_{\alpha} \phi_{\beta}\right]$. (b) Renormalization of the interaction vertex. 
(a)
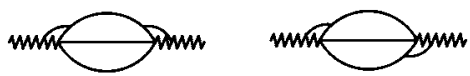

(b)
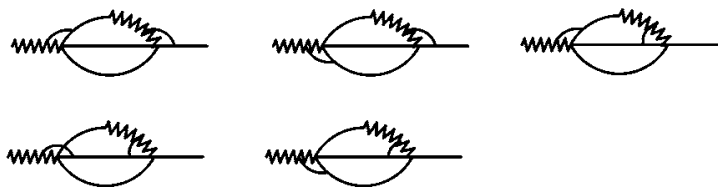

FIG. 6. Feynman diagrams contributing to the renormalization of the propagator to two-loop order in perturbation theory. (a) Contribution to the renormalization of the noise amplitude parameter $D\left(Z_{\omega} Z_{\tilde{\phi}}-1\right)$ in the counterterms $\delta S$ in Eq. (64). (b) Contribution to the renormalization of the other counterterms to the propagator.

$$
\gamma_{\alpha \beta}^{0}(\mathbf{q}, \omega)=\frac{1}{\Delta}\left(\begin{array}{cc}
-i \omega+c \mathbf{q}^{2} & c_{a} \mathbf{q}^{2} \\
-c_{a} \mathbf{q}^{2} & -i \omega+c \mathbf{q}^{2}
\end{array}\right),
$$

where $\Delta=\left(-i \omega+c \mathbf{q}^{2}\right)^{2}+\left(c_{a} \mathbf{q}^{2}\right)^{2}$. The interaction vertex reads

$$
-U_{\alpha \beta} \delta_{\gamma \delta}=-\mu^{\epsilon}(4 \pi)^{-\epsilon / 2} g_{\alpha \beta} \delta_{\gamma \delta},
$$

where $g_{\alpha \beta}$ is given by Eq. (66). Graphic representations of these elements are displayed in Fig. 4. Due to the presence of the nondiagonal element $u_{a}$ in the matrix $U_{\alpha \beta}$, the interaction vertex contains three nonequivalent types of external "legs." The symbol used for the interaction vertex in Fig. 4 indicates this fact. The expressions of the counterterms of the theory are directly visible on the decomposed expressions (64) and (65) of the action of the theory.

Figure 5 displays the diagrams that contribute to the renormalization of the "mass operator" $\left[\widetilde{\phi}_{\alpha} \phi_{\beta}\right]$ and the vertex to one-loop order, and Fig. 6 shows the diagrams that contribute to the renormalization of the propagator to twoloop order in perturbation theory.

\section{APPENDIX B: EXPLICIT EXPRESSIONS OF THE CALCULATED PERTURBATION SERIES}

We present here explicit expressions of the Feynman integrals associated with the diagrams displayed previously, to one- and two-loop order in perturbation theory. Using the notation introduced in Sec. VI A, we denote by $\Gamma_{\left\{\alpha_{i}\right\},\left\{\beta_{j}\right\}}^{(\tilde{N}, N)(n)}$ the contribution of the $n$-loop order to the vertex function with $\tilde{N}$ and $N$ truncated external legs corresponding, respectively, to the fields $\widetilde{\phi}_{\alpha_{i}}$ and $\phi_{\beta_{j}}$, and with $L$ insertions of the mass operator $\left[\tilde{\phi}_{\alpha} \phi_{\beta}\right]$.

\section{One-loop order}

To one-loop order in perturbation theory, the propagator of the theory is not renormalized. The renormalization of the "mass operator" $\left[\widetilde{\phi}_{\alpha} \phi_{\beta}\right]$ is given by

$$
\Gamma_{\alpha, \beta}^{(1,1,1)(1)}=r \mu^{\epsilon}(4 \pi)^{-\epsilon / 2} J_{d}\left[g_{\beta \gamma} \delta_{\alpha \sigma}+g_{\beta \sigma} \delta_{\alpha \gamma}+g_{\beta \alpha} \delta_{\gamma \sigma}\right] \delta_{\gamma \sigma},
$$

where $g_{\alpha \beta}$ is given by Eq. (66). The expression of the integral $J_{d}$ is given below. Because of phase-invariance symmetry, described for $\Gamma_{\alpha, \beta}^{(1,1,1)(1)}$ by Eq. (6), only two terms need to be calculated.

The renormalization of the vertex function to one-loop order in perturbation theory at a symmetry point of the configuration of external momenta respects the following symmetry:

$$
\begin{aligned}
\Gamma_{\alpha_{1}, \beta_{1} \beta_{2} \beta_{3}}^{(1,3,0)(1)}\left(k_{1}, k_{2}, k_{2}, k_{2}\right) \\
=\left[M_{\alpha_{1} \beta_{1}} \delta_{\beta_{2} \beta_{3}}+M_{\alpha_{1} \beta_{2}} \delta_{\beta_{1} \beta_{3}}+M_{\alpha_{1} \beta_{3}} \delta_{\beta_{1} \beta_{2}}\right] \\
\quad \times(2 \pi)^{d+1} \delta^{d+1}\left(k_{1}+3 k_{2}\right),
\end{aligned}
$$

where $M_{\alpha \beta}$ has the phase-invariance symmetry (6). Therefore, the renormalized interaction vertex of the theory remains of the same structure as the original one, and only two independent terms need to be calculated, e.g., $\Gamma_{1,111}^{(1,3,0)(1)}$ and $\Gamma_{2,111}^{(1,3,0)(1)}$. For vanishing external frequencies, we get

$$
\begin{aligned}
\Gamma_{1,111}^{(1,3,0)(1)}= & 24 \mu^{2 \epsilon}(4 \pi)^{-\epsilon}\left[g^{2}\left(\frac{1}{2} I_{d}+2 J_{d}\right)+g_{a}^{2}\left(-\frac{1}{2} I_{d}\right)\right. \\
& \left.+2 g g_{a}\left(\frac{i}{2} I_{d}+i J_{d}\right)+\text { c.c. }\right], \\
\Gamma_{2,111}^{(1,3,0)(1)}= & 24 \mu^{2 \epsilon}(4 \pi)^{-\epsilon}\left[g^{2}\left(-\frac{i}{2} I_{d}\right)+g_{a}^{2}\left(\frac{i}{2} I_{d}+2 i J_{d}\right)\right. \\
& \left.+2 g g_{a}\left(\frac{1}{2} I_{d}+J_{d}\right)+\text { c.c. }\right],
\end{aligned}
$$

where "c.c." denotes the complex conjugate value.

In the previous expressions, the integrals $I_{d}$ and $J_{d}$ are given by

$$
\begin{gathered}
I_{d}\left(c, c_{a}, \mathbf{q}\right)=\int_{\mathbf{p}} \frac{D}{c \mathbf{p}^{2}\left(c+i c_{a}\right)\left[\mathbf{p}^{2}+(\mathbf{p}-\mathbf{q})^{2}\right]} \\
J_{d}\left(c, c_{a}, \mathbf{q}\right)=\int_{\mathbf{p}} \frac{D}{c \mathbf{p}^{2}\left[\left(c-i c_{a}\right) \mathbf{p}^{2}+\left(c+i c_{a}\right)(\mathbf{p}-\mathbf{q})^{2}\right]}
\end{gathered}
$$

Within a dimensional regularization scheme, they read

$$
\begin{aligned}
I_{d}\left(c, c_{a}, \mathbf{q}\right) & =\frac{D}{c\left(c+i c_{a}\right)} \frac{q^{d-4}}{(4 \pi)^{d / 2}} \Gamma\left(\frac{4-d}{2}\right) \int_{0}^{1} \frac{x^{(d-4) / 2}}{(1+x)^{d-2}} d x \\
& =\frac{D}{c\left(c+i c_{a}\right)} \frac{1}{(4 \pi)^{2}(4 \pi)^{-\epsilon / 2}} \frac{1}{\epsilon}[1+O(\epsilon)],
\end{aligned}
$$




$$
\begin{aligned}
J_{d}\left(c, c_{a}, \mathbf{q}\right)= & \frac{D}{c} \frac{q^{d-4}}{(4 \pi)^{d / 2}} \Gamma\left(\frac{d}{2}\right) \Gamma\left(\frac{4-d}{2}\right) \\
& \times \int_{0}^{1} \frac{d x}{(1-x+2 c x)^{2}}\left[x\left(c+i c_{a}\right)\right. \\
& \left.\times \frac{1-x+x\left(c-i c_{a}\right)}{(1-x+2 c x)^{2}}\right]^{(d-4) / 2} \\
= & \frac{D}{c^{2}} \frac{1}{(4 \pi)^{2}(4 \pi)^{-\epsilon / 2}} \frac{1}{\epsilon}[1+O(\epsilon)],
\end{aligned}
$$

where $q=|\mathbf{q}|$.

\section{Explicit expressions of the Feynman integrals to two-loop order}

Since we are looking for the first nontrivial corrections to the critical behaviors in perturbation theory, we only need here to renormalize the propagator, which to one-loop order was not renormalized. Following the same notations as previously, and for vanishing external frequencies, we have

$$
\begin{aligned}
\Gamma_{\alpha_{1} \alpha_{2}}^{(2,0,0)(2)}(\mathbf{k})= & \mu^{2 \epsilon}(4 \pi)^{-\epsilon}\left(g^{2}+g_{a}^{2}\right)\left[2 I_{A}+6 I_{B}\right] \delta_{\alpha \beta}, \\
\Gamma_{1,1}^{(1,1,0)(2)}(\mathbf{k})= & 2 \mu^{2 \epsilon}(4 \pi)^{-\epsilon}\left[g^{2}\left(4 J_{A}+7 J_{C}+J_{E}\right)\right. \\
& \left.+g_{a}^{2}\left(4 J_{A}-7 J_{C}-J_{E}\right)+2 g g_{a}\left(7 J_{D}-J_{F}\right)\right], \\
\Gamma_{2,1}^{(1,1,0)(1)}(\mathbf{k}, 0)= & 2 \mu^{2 \epsilon}(4 \pi)^{-\epsilon}\left[g^{2}\left(-4 J_{B}-7 J_{D}+J_{F}\right)\right. \\
& \left.+g_{a}^{2}\left(-4 J_{B}+7 J_{D}-J_{F}\right)+2 g g_{a}\left(7 J_{C}+J_{E}\right)\right] .
\end{aligned}
$$

$$
\begin{aligned}
& I_{A}=\frac{1}{2}\left[I_{d, \epsilon^{\prime} \epsilon^{\prime \prime}}\left(c_{a}\right)+I_{d, \epsilon^{\prime} \epsilon^{\prime \prime}}\left(-c_{a}\right)\right], \quad \epsilon^{\prime}=+1, \quad \epsilon^{\prime \prime}=-1, \\
& I_{B}=\frac{1}{2}\left[I_{d, \epsilon^{\prime} \epsilon^{\prime \prime}}\left(c_{a}\right)+I_{d, \epsilon^{\prime} \epsilon^{\prime \prime}}\left(-c_{a}\right)\right], \quad \epsilon^{\prime}=-1, \quad \epsilon^{\prime \prime}=+1, \\
& J_{A}=\frac{1}{2}\left[J_{d, \epsilon^{\prime} \epsilon^{\prime \prime}}\left(c_{a}\right)+J_{d, \epsilon^{\prime} \epsilon^{\prime \prime}}\left(-c_{a}\right)\right], \quad \epsilon^{\prime}=+1, \quad \epsilon^{\prime \prime}=-1,
\end{aligned}
$$$$
J_{B}=\frac{i}{2}\left[J_{d, \epsilon^{\prime} \epsilon^{\prime \prime}}\left(c_{a}\right)-J_{d, \epsilon^{\prime} \epsilon^{\prime \prime}}\left(-c_{a}\right)\right], \quad \epsilon^{\prime}=+1, \quad \epsilon^{\prime \prime}=-1,
$$$$
J_{C}=\frac{1}{2}\left[J_{d, \epsilon^{\prime} \epsilon^{\prime \prime}}\left(c_{a}\right)+J_{d, \epsilon^{\prime} \epsilon^{\prime \prime}}\left(-c_{a}\right)\right], \quad \epsilon^{\prime}=-1, \quad \epsilon^{\prime \prime}=+1,
$$$$
J_{D}=\frac{i}{2}\left[J_{d, \epsilon^{\prime} \epsilon^{\prime \prime}}\left(c_{a}\right)-J_{d, \epsilon^{\prime} \epsilon^{\prime \prime}}\left(-c_{a}\right)\right], \quad \epsilon^{\prime}=-1, \quad \epsilon^{\prime \prime}=+1,
$$$$
J_{E}=\frac{1}{2}\left[J_{d, \epsilon^{\prime} \epsilon^{\prime \prime}}\left(c_{a}\right)+J_{d, \epsilon^{\prime} \epsilon^{\prime \prime}}\left(-c_{a}\right)\right], \quad \epsilon^{\prime}=-1, \quad \epsilon^{\prime \prime}=-1,
$$$$
J_{F}=\frac{i}{2}\left[J_{d, \epsilon^{\prime} \epsilon^{\prime \prime}}\left(c_{a}\right)-J_{d, \epsilon^{\prime} \epsilon^{\prime \prime}}\left(-c_{a}\right)\right], \quad \epsilon^{\prime}=-1, \quad \epsilon^{\prime \prime}=-1,
$$

Here

$$
\begin{aligned}
& I_{d, \epsilon^{\prime} \epsilon^{\prime \prime}}\left(c, c_{a}, \mathbf{k}\right)=\int_{\mathbf{p}, \mathbf{q}} \frac{D^{3}}{c \mathbf{q}^{2} c(\mathbf{p}-\mathbf{q})^{2} c(\mathbf{k}-\mathbf{p})^{2}\left[\left(c+i c_{a}\right) \mathbf{q}^{2}+\left(c+i \epsilon^{\prime} c_{a}\right)(\mathbf{p}-\mathbf{q})^{2}+\left(c+i \epsilon^{\prime \prime} c_{a}\right)(\mathbf{k}-\mathbf{p})^{2}\right]}, \\
& J_{d, \epsilon^{\prime} \epsilon^{\prime \prime}}\left(c, c_{a}, \mathbf{k}, \omega_{k}\right)=\int_{\mathbf{p}, \mathbf{q}} \frac{D^{2}}{c \mathbf{q}^{2} c(\mathbf{p}-\mathbf{q})^{2}\left[\left(c+i c_{a}\right) \mathbf{q}^{2}+\left(c+i \epsilon^{\prime} c_{a}\right)(\mathbf{p}-\mathbf{q})^{2}+\left(c+i \epsilon^{\prime \prime} c_{a}\right)(\mathbf{k}-\mathbf{p})^{2}+i \omega_{k}\right]} .
\end{aligned}
$$

The expressions of these integrals as a function of the space dimension $d$ are too large to be displayed here. We therefore just report the expressions of their divergent parts as $\epsilon$ goes to zero,

$$
\begin{gathered}
I_{d, \epsilon^{\prime} \epsilon^{\prime \prime}}\left(c, c_{a}, \mathbf{k}\right)=\frac{D^{3}}{c^{4}} \frac{1}{(4 \pi)^{4}(4 \pi)^{-\epsilon}} \frac{1}{\epsilon} H_{\epsilon^{\prime} \epsilon^{\prime \prime}}\left(\bar{c}_{a}\right)[1+O(\epsilon)], \\
\frac{\partial J_{d, \epsilon^{\prime} \epsilon^{\prime \prime}}\left(c, c_{a}, \mathbf{k}, \omega_{k}\right)}{\partial c \mathbf{k}^{2}} \\
=-\frac{D^{2}}{c^{4}} \frac{1}{(4 \pi)^{4}(4 \pi)^{-\epsilon}} \frac{1}{\epsilon} K_{1, \epsilon^{\prime} \epsilon^{\prime \prime}}\left(\bar{c}_{a}\right)[1+O(\epsilon)],
\end{gathered}
$$

$$
\begin{aligned}
& \frac{\partial J_{d, \epsilon^{\prime} \epsilon^{\prime \prime}}\left(c, c_{a}, \mathbf{k}, \omega_{k}\right)}{\partial i \omega_{k}} \\
& =-\frac{D^{2}}{c^{3}} \frac{1}{(4 \pi)^{4}(4 \pi)^{-\epsilon}} \frac{1}{\epsilon} K_{2, \epsilon^{\prime} \epsilon^{\prime \prime}}\left(\bar{c}_{a}\right)[1+O(\epsilon)],
\end{aligned}
$$

where $\bar{c}_{a}=c_{a} / c$. The integrals $H_{\epsilon^{\prime} \epsilon^{\prime \prime}}\left(\bar{c}_{a}\right), K_{1, \epsilon^{\prime} \epsilon^{\prime \prime}}\left(\bar{c}_{a}\right)$, and $K_{2, \epsilon^{\prime} \epsilon^{\prime \prime}}\left(\bar{c}_{a}\right)$ are given by expressions that are similar to the ones found to the one-loop order [Eq. (B5)], which for $d$ $=4$ reduce to integrals of rational fractions with complex parameters. The results of these integrations read 


$$
\begin{gathered}
K_{1,+1+1}\left(\bar{c}_{a}\right)=I_{1}\left(\bar{c}_{a}\right)=\frac{1}{6\left(1+i \bar{c}_{a}\right)}, \\
K_{1,-1-1}\left(\bar{c}_{a}\right)=I_{2}\left(\bar{c}_{a}\right)=\frac{2+i \bar{c}_{a}}{4\left(3+i \bar{c}_{a}\right)}, \\
K_{1,-1+1}\left(\bar{c}_{a}\right)=I_{3}\left(\bar{c}_{a}\right)=I_{2}\left(-\bar{c}_{a}\right), \\
K_{1,+1-1}\left(\bar{c}_{a}\right)=I_{4}\left(\bar{c}_{a}\right)=\frac{1-i \bar{c}_{a}}{6-2 i \bar{c}_{a}}, \\
K_{2,+1+1}\left(\bar{c}_{a}\right)=I_{5}\left(\bar{c}_{a}\right)=\frac{1}{\left(1+i \bar{c}_{a}\right)^{2}} \ln \left[\frac{4}{3}\right], \\
K_{2,-1-1}\left(\bar{c}_{a}\right)=I_{6}\left(\bar{c}_{a}\right)=\frac{1}{\left(1-i \bar{c}_{a}\right)^{2}} \ln \left[\frac{4}{3+i \bar{c}_{a}}\right], \\
K_{2,-1+1}\left(\bar{c}_{a}\right)=I_{7}\left(\bar{c}_{a}\right)=I_{6}\left(-\bar{c}_{a}\right), \\
\left(\bar{c}_{a}\right)=H_{-1+1}\left(\bar{c}_{a}\right)=I_{9}\left(\bar{c}_{a}\right), \\
\left.\left(\bar{c}_{a}\right)=I_{8}\left(\bar{c}_{a}\right)=\frac{4}{\left(1-i \bar{c}_{a}\right)\left(1+i \bar{c}_{a}\right)}\right], \\
\ln \left[\frac{4}{(3-1-1}\right]
\end{gathered}
$$

where $I_{9}\left(\bar{c}_{a}\right)$ can be represented by

$$
\begin{aligned}
I_{9}\left(\bar{c}_{a}\right)= & \frac{1}{\left(1+\bar{c}_{a}^{2}\right)}\left\{4 \ln [2]-i \arctan \left[\frac{2 \bar{c}_{a}}{3+\bar{c}_{a}^{2}}\right]\right. \\
& -\frac{1}{2} \ln \left[9+10 \bar{c}_{a}^{2}+\bar{c}_{a}^{4}\right]-\ln \left[-i\left(1+2 i \bar{c}_{a}+\bar{c}_{a}^{2}\right)\right] \\
& \left.+\ln \left[-i \frac{1+2 i \bar{c}_{a}+\bar{c}_{a}^{2}}{3+2 i \bar{c}_{a}+\bar{c}_{a}^{2}}\right]\right\} \\
& -\frac{1}{\sqrt{\left(1+i \bar{c}_{a}\right)^{2}}}\left\{\ln \left[-i \frac{\sqrt{\left(1+i \bar{c}_{a}\right)^{2}}\left(1-i \bar{c}_{a}\right)}{\left[1+i \bar{c}_{a}+\sqrt{\left(1+i \bar{c}_{a}\right)^{2}}\right]^{2}}\right]\right. \\
& \left.-\ln \left[-i \frac{2+i \bar{c}_{a}+\bar{c}_{a}^{2}-\sqrt{\left(1+i \bar{c}_{a}\right)^{2}}}{2+i \bar{c}_{a}+\bar{c}_{a}^{2}+\sqrt{\left(1+i \bar{c}_{a}\right)^{2}}}\right]\right\}
\end{aligned}
$$

Because of the ambiguity in the definition of the complex logarithm, the integrals $I_{6}\left(\bar{c}_{a}\right), I_{7}\left(\bar{c}_{a}\right), I_{8}\left(\bar{c}_{a}\right)$, and $I_{9}\left(\bar{c}_{a}\right)$ are not uniquely defined by these expressions [55]. To specify entirely these functions, one needs to use their values for $\bar{c}_{a}=0$, which read

$$
\begin{gathered}
I_{6}\left(\bar{c}_{a}=0\right)=I_{7}\left(\bar{c}_{a}=0\right)=I_{8}\left(\bar{c}_{a}=0\right)=\ln \left[\frac{4}{3}\right], \\
I_{9}\left(\bar{c}_{a}=0\right)=3 \ln \left[\frac{4}{3}\right] .
\end{gathered}
$$

The entire functions are then defined as the unique analytic prolongations of the expressions (B10) and (B11), defined in the vicinity of $\bar{c}_{a}=0$ together with the specification (B12).

\section{APPENDIX C: EXPLICIT EXPRESSIONS OF THE RENORMALIZATION GROUP EQUATIONS}

With units such that $c=1$, the function $\beta_{c}\left(g, g_{a}, c_{a}\right)$ is given by

$$
\begin{aligned}
\beta_{c}\left(g, g_{a}, c_{a}\right)= & \mathcal{D}^{2}\left[\left(g^{2}+g_{a}^{2}\right) \operatorname{Im}\left(-I_{4}\right)\right. \\
& \left.+\left(g^{2}-g_{a}^{2}\right) \operatorname{Im}\left(2 I_{2}\right)+2 g g_{a} \operatorname{Re}\left(-2 I_{2}\right)\right] \\
& +\mathcal{D}^{2} \bar{c}_{a}\left[\left(g^{2}+g_{a}^{2}\right) \operatorname{Re}\left(I_{4}\right)+\left(g^{2}-g_{a}^{2}\right)\right. \\
& \left.\times \operatorname{Re}\left(2 I_{2}\right)+2 g g_{a} \operatorname{Im}\left(2 I_{2}\right)\right]+\mathcal{D}^{2} c_{a}\left(c_{a}+c_{a}^{-1}\right) \\
& \times\left[\left(g^{2}+g_{a}^{2}\right) \operatorname{Im}\left(I_{8}\right)+\left(g^{2}-g_{a}^{2}\right) \operatorname{Im}\left(-2 I_{6}\right)\right. \\
& \left.+2 g g_{a} \operatorname{Re}\left(2 I_{6}\right)\right],
\end{aligned}
$$

where $\mathcal{D}=4 D /(4 \pi)^{2}$ [56]. The function $\rho_{c}\left(c_{a}\right)$, which is defined in Eq. (74) and determines the fixed points of the theory to two-loop order, is given by

$$
\begin{aligned}
\rho_{c}\left(c_{a}\right)= & -\frac{1}{50}\left(1+c_{a}^{2}\right)\left[4\left(1-c_{a}^{2}\right) \operatorname{Im}\left[\frac{\ln \left(\frac{4}{3+i c_{a}}\right)}{\left(1-i c_{a}\right)^{2}}\right]\right. \\
& -2\left(1+c_{a}^{2}\right) \operatorname{Im}\left[\frac{\ln \left(\frac{4}{3+2 i c_{a}+c_{a}^{2}}\right)}{\left(1-i c_{a}\right)^{2}}\right] \\
& \left.-8 c_{a} \operatorname{Re}\left[\frac{\left.\left.\ln \left(\frac{4}{3+i c_{a}}\right)\right]\right\}}{\left(1-i c_{a}\right)^{2}}\right]\right\}
\end{aligned}
$$

where the three complex logarithms are defined by prolongation of their real values for $c_{a}=0$. This function is displayed in Fig. 2.

Finally, the other Wilson's functions of the theory are given by the following expressions:

$$
\begin{aligned}
\gamma_{\theta}\left(g, g_{a}, c_{a}\right)= & \mathcal{D}^{2}\left[\left(g^{2}+g_{a}^{2}\right) \operatorname{Im}\left(I_{8}\right)+\left(g^{2}-g_{a}^{2}\right) \operatorname{Im}\left(-2 I_{6}\right)\right. \\
& \left.+2 g g_{a} \operatorname{Re}\left(2 I_{6}\right)\right],
\end{aligned}
$$

and

$$
\begin{aligned}
\gamma\left(g, g_{a}, c_{a}\right)= & \mathcal{D}^{2}\left[\left(g^{2}+g_{a}^{2}\right) \operatorname{Re}\left(3 I_{4}-I_{8}-I_{9}\right)\right. \\
+ & \left.\left(g^{2}-g_{a}^{2}\right) \operatorname{Re}\left(6 I_{2}-2 I_{6}\right)+2 g g_{a} \operatorname{Im}\left(6 I_{2}-2 I_{6}\right)\right] \\
+ & 3 \mathcal{D}^{2} c_{a}\left[\left(g^{2}+g_{a}^{2}\right) \operatorname{Im}\left(I_{8}\right)\right. \\
+ & \left.\left(g^{2}-g_{a}^{2}\right) \operatorname{Im}\left(-2 I_{6}\right)+2 g g_{a} \operatorname{Re}\left(2 I_{6}\right)\right] \\
\gamma_{\omega}\left(g, g_{a}, c_{a}\right)= & \mathcal{D}^{2}\left[\left(g^{2}+g_{a}^{2}\right) \operatorname{Re}\left(-I_{4}+I_{8}\right)\right. \\
& +\left(g^{2}-g_{a}^{2}\right) \operatorname{Re}\left(-2 I_{2}+2 I_{6}\right) \\
& \left.+2 g g_{a} \operatorname{Im}\left(-2 I_{2}+2 I_{6}\right)\right] \\
& -\mathcal{D}^{2} c_{a}\left[\left(g^{2}+g_{a}^{2}\right) \operatorname{Im}\left(I_{8}\right)+\left(g^{2}-g_{a}^{2}\right) \operatorname{Im}\left(-2 I_{6}\right)\right. \\
& \left.+2 g g_{a} \operatorname{Re}\left(2 I_{6}\right)\right],
\end{aligned}
$$




$$
\begin{aligned}
\widetilde{\gamma}\left(g, g_{a}, c_{a}\right)= & \mathcal{D}^{2}\left[\left(g^{2}+g_{a}^{2}\right) \operatorname{Re}\left(I_{4}-I_{8}+I_{9}\right)\right. \\
& \left.+\left(g^{2}-g_{a}^{2}\right) \operatorname{Re}\left(2 I_{2}-2 I_{6}\right)+2 g g_{a} \operatorname{Im}\left(2 I_{2}-2 I_{6}\right)\right] \\
& +\mathcal{D}^{2} c_{a}\left[\left(g^{2}+g_{a}^{2}\right) \operatorname{Im}\left(I_{8}\right)\right. \\
& \left.+\left(g^{2}-g_{a}^{2}\right) \operatorname{Im}\left(-2 I_{6}\right)+2 g g_{a} \operatorname{Re}\left(2 I_{6}\right)\right] . \quad(\mathrm{C} 4)
\end{aligned}
$$

\section{APPENDIX D: GINZBURG CRITERION}

The critical behaviors described by the RG fixed point are valid in the proximity of the critical point. In practice, one can estimate how close to the critical point an experiment needs to be performed in order for the critical behaviors to become observable. Further away from the critical point, when mean-field theory is still an appropriate approximation, nontrivial critical exponents are unobservable. The Ginzburg criterion estimates the breakdown of mean-field theory at the point where the variance of order-parameter fluctuations exceeds its average [50]. For simplicity, we discuss here the real equation (2) with $u_{a}=0$ and $c_{a}=0$. Based on this criterion, mean field theory is valid if

$$
\frac{D \xi^{(d-2)}}{c}<\frac{|r|}{u}
$$

with $\xi^{2}=c /|r|$. We now relate this expression to a bulk chemical system where oscillations occur if the concentra- tion $\rho$ of some species exceeds a critical value $\rho_{c}: r \simeq a\left(\rho_{c}\right.$ $-\rho$ ), where $a$ is a proportionality coefficient. In this case, the real part of $Z$ is related to the concentration fluctuations $\delta \rho / \rho$. As a consequence, $r$ and $u$ have dimensions of an inverse time, while the noise strength $D$ is a volume per unit of time. The coefficient $c$ has units of a diffusion coefficient. We can estimate the fluctuations of the number $N$ of molecules in a reference volume $v$ after a time $\tau$ using $\partial_{t} \delta \rho \simeq \rho \eta$ as

$$
\left\langle\delta N^{2}\right\rangle \simeq \int_{v} d^{d} x d^{d} x^{\prime}\left\langle\delta \rho(\mathbf{x}) \delta \rho\left(\mathbf{x}^{\prime}\right)\right\rangle \simeq \rho^{2} D v \tau .
$$

We estimate $\left\langle\delta N^{2}\right\rangle / N^{2} \simeq 1 / N \simeq 1 / l_{c}^{3} \rho$ within a reaction volume $v=l_{c}^{3}$ and a reaction time $\tau_{c}$, with $l_{c}^{2}=D_{m} \tau_{c}$ and where $D_{m}$ is a microscopic diffusion coefficient. From this, it follows that $D \simeq 1 /\left(\rho \tau_{c}\right)$. For $d=3$, Eq. (D1) implies that meanfield theory is valid if

$$
\frac{\left|\rho-\rho_{c}\right|}{\rho_{c}}>\frac{u^{2} D^{2}}{r_{0} c^{3}}
$$

where $r_{0}=a \rho_{c}$. Estimating $r_{0} \sim u \sim \omega_{0}$, we find Eqs. (88) and (89).
[1] M. E. Fisher, Rep. Prog. Phys. 30, 615 (1967).

[2] E. Brézin, J. C. Le Guillou, and J. Zinn-Justin, in Phase Transitions and Critical Phenomena, edited by C. Domb and M. S. Green (Academic Press, London, 1976), Vol. 6, pp. 125-247.

[3] P. C. Hohenberg and B. I. Halperin, Rev. Mod. Phys. 49, 435 (1977).

[4] L. P. Kadanoff et al., Rev. Mod. Phys. 39, 395 (1967).

[5] K. G. Wilson, Phys. Rev. B 4, 3174 (1971).

[6] K. G. Wilson, Phys. Rev. B 4, 3184 (1971).

[7] K. G. Wilson and J. Kogut, Phys. Rep., Phys. Lett. 12, 75 (1974).

[8] K. G. Wilson, Rev. Mod. Phys. 47, 773 (1975).

[9] C. De Dominicis and L. Peliti, Phys. Rev. B 18, 353 (1978).

[10] J. B. Kogut, Rev. Mod. Phys. 51, 659 (1979).

[11] K. G. Wilson, Rev. Mod. Phys. 55, 583 (1983).

[12] M. E. Fisher, Rev. Mod. Phys. 70, 653 (1998).

[13] J. Zinn-Justin, Quantum Field Theory and Critical Phenomena (Oxford University Press, New York, 1996).

[14] B. Schmittmann and R. K. P. Zia, in Phase Transitions and Critical Phenomena, edited by C. Domb and J. L. Lebowitz (Academic Press, London, 1995), Vol. 17.

[15] D. S. Fisher, Phys. Rep. 301, 113 (1998).

[16] H. Hinrichsen, Adv. Phys. 49, 815 (2000).

[17] U. C. Täuber, V. K. Akkineni, and J. E. Santos, Phys. Rev. Lett. 88, 045702(1) (2002).

[18] E. Medina, T. Hwa, M. Kardar, and Y.-C. Zhang, Phys. Rev. A 39, 3053 (1989).

[19] E. Frey and U. C. Täuber, Phys. Rev. E 50, 1024 (1994).

[20] E. Frey, U. C. Täuber, and F. Schwabl, Phys. Rev. E 49, 5058 (1994).
[21] H.-K. Janssen, Phys. Rev. Lett. 78, 1082 (1997).

[22] Y. Y. Goldschmidt, H. Hinrichsen, M. Howard, and U. C. Täuber, Phys. Rev. E 59, 6381 (1999).

[23] T. Risler, J. Prost, and F. Jülicher, Phys. Rev. Lett. 93, 175702(1) (2004).

[24] S. H. Strogatz, Nonlinear Dynamics and Chaos (Perseus Books Publishing, Cambridge, MA, 2000).

[25] P. Bergé, Y. Pomeau, and C. Vidal, Order Within Chaos (Hermann, Paris, 1984).

[26] S. Camalet, T. Duke, F. Jülicher, and J. Prost, Proc. Natl. Acad. Sci. U.S.A. 97, 3183 (2000).

[27] V. M. Eguíluz, M. Ospeck, Y. Choe, A. J. Hudspeth, and M. O. Magnasco, Phys. Rev. Lett. 84, 5232 (2000).

[28] A. C. Newell and J. A. Whitehead, in Instability of Continuous Systems, edited by H. H. E. Leipholz (Springer-Verlag, Berlin, 1971), p. 284.

[29] I. S. Aranson and L. Kramer, Rev. Mod. Phys. 74, 99 (2002).

[30] P. C. Martin, E. D. Siggia, and H. A. Rose, Phys. Rev. A 8, 423 (1973).

[31] H.-K. Janssen, Z. Phys. B 23, 377 (1976).

[32] C. De Dominicis, J. Phys. (Paris), Colloq. 1, C-247 (1976).

[33] E. Brézin, J. C. Le Guillou, and J. Zinn-Justin, Phys. Rev. B 10, 892 (1974).

[34] R. Bausch, H. K. Janssen, and H. Wagner, Z. Phys. B 24, 113 (1976).

[35] U. C. Täuber and F. Schwabl, Phys. Rev. B 46, 3337 (1992).

[36] K. G. Wilson and M. E. Fisher, Phys. Rev. Lett. 28, 240 (1972).

[37] K. G. Wilson, Phys. Rev. D 7, 2911 (1973).

[38] J. Rudnick and D. R. Nelson, Phys. Rev. B 13, 2208 (1976). 
[39] P. Martin, A. J. Hudspeth, and F. Jülicher, Proc. Natl. Acad. Sci. U.S.A. 98, 14380 (2001).

[40] F. Haake, M. Lewenstein, and M. Wilkens, Z. Phys. B: Condens. Matter 55, 211 (1984).

[41] G. Grinstein, C. Jayaprakash, and Y. He, Phys. Rev. Lett. 55, 2527 (1985).

[42] K. E. Bassler and B. Schmittmann, Phys. Rev. Lett. 73, 3343 (1994).

[43] U. C. Täuber and Z. Rácz, Phys. Rev. E 55, 4120 (1997).

[44] J. M. Kosterlitz and D. J. Thouless, J. Phys. C 6, 1181 (1973).

[45] F. Jülicher, A. Ajdari, and J. Prost, Rev. Mod. Phys. 69, 1269 (1997).

[46] R. Kapral and K. Showalter, Chemical Waves and Patterns (Kluwer, Dordrecht, 1995).

[47] H. A. Braun, H. Wissing, K. Schäfer, and M. C. Hirsch, Nature (London) 367, 270 (1994).

[48] A. Neiman and D. F. Russell, Phys. Rev. Lett. 86, 3443 (2001).

[49] M. Ospeck, V. M. Eguíluz, and M. O. Magnasco, Biophys. J. 80, 2597 (2001).

[50] V. L. Ginzburg, Sov. Phys. Solid State 2, 1824 (1960).
[51] Note that because of space-time translational invariance of the theory, these functions depend only on the differences $\mathbf{x}-\mathbf{x}^{\prime}$ and $t-t^{\prime}$.

[52] Note that we do not write the Jacobian term that appears in general in this formalism. Its role is indeed to compensate loops $\left\langle\psi_{\alpha} \widetilde{\psi}_{\beta}\right\rangle_{0}$ that begin and end at the same vertex in a perturbative development. This choice, followed by the prescription that such self-loops vanish, is consistent and preserves causality [13].

[53] Note that $G_{11}>0$ so that $F$ can always be defined.

[54] The divergence of the effective temperature for small $q$ follows from simple scaling arguments. The divergence for small $\omega$ $-\omega_{0}^{\text {eff }}$, however, results from the nontrivial renormalization of $\omega_{0}$ as described by Eqs. (80) and (81).

[55] Note that, because of this ambiguity, the expression (B11) cannot be simplified further.

[56] Note that the integral $I_{9}$ does not enter the expression of the beta function here. Therefore, this quantity is not affecting the determination of the fixed points to this order in perturbation theory. 